\title{
Computational Design of Molecular Motors and Excited-State Studies of Organic Chromophores
}

\author{
Baswanth Oruganti
}

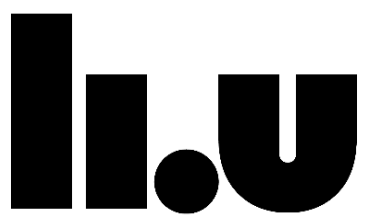

LINKÖPING UNIVERSITY

Department of Physics, Chemistry, and Biology (IFM)

Linköping University, SE-581 83 Linköping, Sweden

Linköping 2016 
(C)Baswanth Oruganti, 2016

Printed in Sweden by LiU-Tryck, Linköping, Sweden, 2016

ISBN 978-91-7685-674-1

ISSN 0345-7524 
To my Loving Dad

నాన్నకు ప్రేమతో అంకితం 


\section{Abstract}

This thesis presents computational quantum chemical studies of molecular motors and excited electronic states of organic chromophores.

The first and major part of the thesis is concerned with the design of lightdriven rotary molecular motors. These are molecules that absorb light energy and convert it into $360^{\circ}$ unidirectional rotary motion around a double bond connecting two molecular halves. In order to facilitate potential applications of molecular motors in nanotechnology, such as in molecular transport or in development of materials with photo-controllable properties, it is critical to optimize the rates and efficiencies of the chemical reactions that produce the rotary motion. To this end, computational methods are in this thesis used to study two different classes of molecular motors.

The first class encompasses the sterically overcrowded alkenes developed by Ben Feringa, co-recipient of the 2016 Nobel Prize in Chemistry. The rotary cycles of these motors involve two photoisomerization and two thermal isomerization steps, where the latter are the ones that limit the attainable rotational frequencies. In the thesis, several new motors of this type are proposed by identifying steric, electronic and conformational approaches to accelerate the thermal isomerizations. The second class contains motors that incorporate a protonated Schiff base and are capable to achieve higher photoisomerization rates than overcrowded alkene-based motors. In the thesis, a new motor of this type is proposed that produces unidirectional rotary motion by means of two photochemical steps alone. Also, this motor lacks both a stereocenter and helical motifs, which are key features of almost all synthetic rotary motors developed to date.

The second part of the thesis focuses on the design and assessment of composite computational procedures for modeling excited electronic states of organic chromophores. In particular, emphasis is put on developing procedures that facilitate the calculations of accurate $0-0$ excitation energies of such compounds in a costeffective way by combining quantum chemical methods with different accuracies. 


\section{Populärvetenskaplig Sammanfattning}

Varje system oavsett storlek som förmår omvandla energi från en yttre källa till användbar mekanisk rörelse såsom rotation kan kallas för en "motor". I människokroppen förekommer naturligt ett stort antal motorer av molekylär storlek som omsätter kemisk energi från födan för att utföra ett flertal viktiga biologiska funktioner, varav muskelkontraktion och intracellulär transport hör till de mest kända. Design och laboratoriesyntes av molekylära motorer som på ett kontrollerat sätt åstadkommer rörelse och kan användas för praktiska ändamål, är dock förenat med avsevärda utmaningar. Trots det utgör fältet en starkt växande gren av nanotekniken, inom vilket en rad framsteg rapporterats under senare år. Tre av fältets förgrundsfigurer belönades också med 2016 års Nobelpris i kemi.

Den första och huvudsakliga delen av denna avhandling utgår från en speciell typ av molekylära motorer som utvecklats av Ben Feringa, en av ovanstående pristagare. Dessa motorer förmår omvandla kontinuerligt tillförd ljusenergi till kontinuerlig 360-gradig rotationsrörelse kring en kemisk bindning, och uppvisar redan i dagsläget en imponerande prestanda såtillvida att de kan nå rotationsfrekvenser i MHz-området (d.v.s., de kan rotera miljontals varv per sekund). Men för att fullt ut dra nytta av dessa och andra motorers potential att fungera som effektiva kraftkällor i olika sammanhang, t.ex. för transport och målspecifik leverans av läkemedel i kroppen, är det emellertid av största vikt att ytterligare förbättra befintliga motorers prestanda, samt även att designa helt nya motorer som har mer fördelaktiga egenskaper. I avhandlingen presenteras forskning där flera möjligheter att möta dessa mål undersöks med olika beräkningsmetoder inom teoretisk kemi.

Avhandlingens andra del handlar också om ljusabsorberande molekyler, s.k. kromoforer, men fokuserar på design och utvärdering av nya tillvägagångssätt för att beskriva exciterade energitillstånd hos sådana molekyler mer noggrant, utan att nödvändigtvis ta till kostsamma beräkningsmetoder. Speciellt undersöks möjligheten att på detta sätt beskriva organiska kromoforer av en typ som är relevant för solceller och lysdioder. 


\section{Acknowledgements}

First of all, I would like to express my deep sense of gratitude to my $\mathrm{PhD}$ supervisor Bo Durbeej for his invaluable guidance, thought provoking discussions and critical evaluation of my work with immense care and patience. Thanks a lot Bo, for constantly pushing me to perform better.

I am very thankful to Changfeng Fang and Jun Wang, not only for their significant contributions in the design and execution of research projects but also for being friendly and cooperative. I thank Olle Falklöf for his help in all practical things both in and out of work.

I would like to thank Patrick Norman for his inspirational leadership of our group, and for sponsoring me to attend the Molecular Response Properties Winter School at Luchon. My special thanks to Mathieu Linares for being very friendly, and for introducing me to molecular dynamics. I am thankful to Iryna Yakymenko for teaching four different courses, and particularly for the courses in quantum computers and mathematical methods in physics.

I am grateful to Lejla Kronbäck for her quick help in all administrative issues. I thank Jessica Gidby for her suggestions regarding issues with residence permit. My great thanks to all present and former colleagues in the Theoretical Chemistry group (formerly Computational Physics group), particularly Riccardo Volpi, Jonas Björk and Paulo Medeiros for being friendly. Thanks a lot to my friends Anil, Sravan and Vinay for constant encouragement and help throughout my $\mathrm{PhD}$.

Coming to my family, I owe a lot to my loving wife who sacrificed many weekend plans for my $\mathrm{PhD}$. My mother and brother have always been supportive and understood me for missing my brother's wedding. I thank them from the bottom of my heart. Finally, the acknowledgments would be incomplete if I don't mention the two most inspiring people in my life: my dad and my chemistry teacher Dr. Bala Karuna Kumar. 


\section{Contents}

1 Introduction 1

2 Computational Methods 5

2.1 Basic Quantum Chemistry 5

2.1.1 Hartree-Fock Theory 8

$\begin{array}{lll}2.1 .2 & \text { Electron Correlation } & 10\end{array}$

$\begin{array}{lll}\text { 2.1.3 Density Functional Theory } & 14\end{array}$

$\begin{array}{lll}2.2 & \text { Modeling Excited Electronic States } & 18\end{array}$

$\begin{array}{lll}2.2 .1 & \text { Single-Configurational Methods } & 18\end{array}$

2.2.2 Multi-Configurational Methods 22

2.3 Non-Adiabatic Molecular Dynamics Simulations 25

2.4 Solvent Effects $\quad 26$

2.4.1 Polarizable Continuum Models 27

3 Light-Driven Rotary Molecular Motors 29

3.1 Sterically Overcrowded Alkenes 29

3.1.1 Paper I 32

3.1.2 Paper II 34

3.1.3 Paper III 36

3.1.4 Paper IV 38

3.2 Protonated Schiff Bases 41

3.2.1 Paper V 42

4 Excited-State Studies of Organic Chromophores 45

4.1 Composite Procedures 45

4.1.1 Paper VI 46

4.1.2 Paper VII 49

5 Concluding Remarks

$\begin{array}{ll}\text { Bibliography } & 57\end{array}$

Publications Included in the Thesis $\quad 63$ 


\section{Introduction}

Nature performs a variety of complex biological tasks using molecular systems that produce controlled mechanical motion by absorbing light or chemical energy. One such task is the process of human vision that involves conversion of light energy into controlled rotation around a chemical bond in the retinal chromophore of the visual pigment rhodopsin. ${ }^{1,2}$ Another example is proton transport across cell membranes through chemically induced $360^{\circ}$ unidirectional rotary motion in ATPase proteins. ${ }^{3,4}$ In general, a molecule capable of producing controlled mechanical motion in a repetitive and progressive manner by absorbing external energy is termed a molecular motor. Molecular motors, such as ATPases, that produce $360^{\circ}$ unidirectional rotary motion are referred to as rotary molecular motors.

The idea of synthetic molecular motors capable of mimicking their biological counterparts was first formulated by Richard Feynman in his 1959 talk There's plenty of room at the bottom. ${ }^{5}$ However, his idea was not realized until the late 1990s. Ben Feringa, co-recipient of the 2016 Nobel Prize in Chemistry, and his coworkers reported the first synthetic molecular motor in $1999 .{ }^{6}$ This motor, shown in Figure 1.1 and hereafter referred to as motor $\mathbf{1}$, absorbs UV light and produces unidirectional rotary motion around a carbon-carbon double bond in a sterically overcrowded alkene. The double bond connects two identical phenanthrylidene moieties and acts as an axle for the rotation of one moiety (rotator) relative to the other, which is surfaceimmobilizable (stator) ${ }^{7-9}$ The motor has two essential chiral features. First, both the stator and rotator halves feature a stereocenter, whose $R$ or $S$ configurations determine the direction - clockwise $(\mathrm{CW})$ or counterclockwise $(\mathrm{CCW})$ - of the photoinduced rotation. ${ }^{6}$ Second, due to the steric interactions between the stator and rotator in the so-called fjord regions, the motor halves are not planar but adapt helical geometries, denoted $P$ or $M$ to indicate right-handed or left-handed helicities, respectively. ${ }^{6}$ 


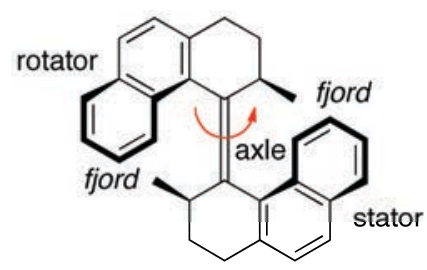

1

Figure 1.1. Chemical structure of the light-driven rotary molecular motor $\mathbf{1}$.

In contrast to Nature's ATPase motor that is capable of achieving 130 rotations per second, ${ }^{3}$ one full $360^{\circ}$ rotation of motor 1 was found to take $\sim 440$ hours under ambient conditions. ${ }^{10}$ In this light, a key requirement for harnessing the mechanical motion produced by molecular motors of type $\mathbf{1}$ in nanotechnology, ${ }^{11-16}$ such as in molecular transport ${ }^{12,13}$ and in development of smart materials with photoswitchable properties, ${ }^{11,14-16}$ is that the motors can achieve much higher rotary rates and efficiencies under ambient conditions. A major experimental effort has therefore been invested to improve the rates and efficiencies of overcrowded alkene-based molecular motors. ${ }^{9,10,17-31}$ In this regard, computational methods are particularly useful as they can provide detailed insights into the mechanisms and dynamics of the chemical reactions that produce the rotary motion. Such insights may not be easily obtained by experimental techniques, because of the difficulties associated with detecting transient species such as reactive intermediates and transition structures (TSs) or measuring the timescales of ultrafast processes.

The first and major part of the present thesis focuses on computational design of light-driven rotary molecular motors. Specifically, the first five papers included in the thesis are devoted to this topic. Among these, the first four deal with improving the performance of the rotary cycles of overcrowded alkene-based molecular motors. The fifth paper proposes a novel Schiff-base molecular motor that, unlike overcrowded alkene-based motors, lacks both stereocenters and helical motifs but achieves unidirectional rotary motion more efficiently.

The second part of the thesis is also concerned with photoactive molecules (chromophores), but mainly considers rigid organic chromophores such as the aromatic hydrocarbons and aromatic heterocyclic compounds shown in Figure 1.2, which are important for applications in organic electronics. ${ }^{32-35}$ Specifically, in this part, composite computational procedures that combine theoretical methods of 
different accuracies are proposed and tested for cost-effective applications to excited states of organic chromophores. The sixth paper of the thesis investigates some prerequisites for such procedures to work and the seventh paper reports the design and assessment of a new composite procedure for cheaper calculations of accurate UV-vis excitation energies.<smiles>c1ccc2ccccc2c1</smiles>

naphthalene<smiles>c1ccc2c(c1)[nH]c1ccccc12</smiles>

9H-carbazole<smiles>c1ccc2cc3ccccc3cc2c1</smiles>

anthracene<smiles></smiles>

perylene<smiles>c1ccc2cc3cc4ccccc4cc3cc2c1</smiles>

tetracene<smiles>C=Nc1cc[nH]c2ccc(c1)cc1nc(cc3nc4cc(ccc(c2)[nH]3)N4)C=C1</smiles>

porphyrin

Figure 1.2. Examples of some organic chromophores studied.

The thesis is organized into five chapters. Chapter 2 discusses the computational methods used for studies of molecular motors and excited states of organic chromophores. Chapter 3 gives a brief background to molecular motors and summarizes the key results of the first five papers. Chapter 4 presents a short introduction to composite procedures for excited-state studies and outlines the major findings of the sixth and seventh papers. Finally, Chapter 5 provides some concluding remarks. 
Introduction 


\section{Computational Methods}

Computational chemistry is the field concerned with the development and application of theoretical methods for studying problems of chemical interest. These methods are based on physical theories that describe the behaviour of matter and its interactions. While methods rooted in classical mechanics are usually employed to simulate the dynamics of atomic motions, they are inadequate for modeling chemical reactions, which involve changes in the electronic structure of the species involved. Methods founded on quantum mechanics, on the other hand, provide a proper description of electrons. At sufficiently small velocities, the behavior of electrons can be treated within the non-relativistic framework of quantum mechanics. Although there are several rigorous mathematical formulations of non-relativistic quantum mechanics, ${ }^{36}$ Schrödinger's wavefunction formalism is the commonly used one in quantum chemistry, and solving the Schrödinger equation is the central focus of most quantum chemical approaches.

\subsection{Basic Quantum Chemistry}

The non-relativistic time-dependent Schrödinger equation for a molecular system is given (in atomic units) by

$$
\hat{H} \Psi(\mathbf{r}, \mathbf{R}, t)=i \frac{\partial \Psi(\mathbf{r}, \mathbf{R}, t)}{\partial t},
$$

where $\Psi(\mathbf{r}, \mathbf{R}, t)$ is the time-dependent wavefunction of the system describing both electronic (r) and nuclear (R) spatial coordinates, and $\hat{H}$ is the Hamiltonian operator of the system. In the absence of any external fields, the Hamiltonian operator of a system containing $N$ electrons and $M$ nuclei is given by

$$
\hat{H}=\hat{T}_{e}+\hat{T}_{n}+\hat{V}_{e n}+\hat{V}_{e e}+\hat{V}_{n n},
$$




$$
\hat{H}=-\frac{1}{2} \sum_{i=1}^{N} \nabla_{i}^{2}-\frac{1}{2} \sum_{A=1}^{M} \frac{1}{M_{A}} \nabla_{A}^{2}-\sum_{i=1}^{N} \sum_{A=1}^{M} \frac{Z_{A}}{\mathbf{r}_{i A}}+\sum_{i=1}^{N} \sum_{j>i}^{N} \frac{1}{\mathbf{r}_{i j}}+\sum_{A=1}^{M} \sum_{B>A}^{M} \frac{Z_{A} Z_{B}}{\mathbf{r}_{A B}} .
$$

Here, the first two terms correspond to the kinetic energies of electrons and nuclei, and the last three terms describe the potential energies of electron-nucleus, electronelectron and nucleus-nucleus interactions, respectively. As the Hamiltonian in Eq. (2.3) is time-independent, the time-dependence of the wavefunction appears merely as an exponential prefactor,

$$
\Psi(\mathbf{r}, \mathbf{R}, t)=e^{-i E t} \Psi(\mathbf{r}, \mathbf{R}),
$$

where $E$ is the energy characterizing a stationary state of the system. Substituting Eq. (2.4) in Eq. (2.1) yields the time-independent Schrödinger equation

$$
\hat{H} \Psi(\mathbf{r}, \mathbf{R})=E \Psi(\mathbf{r}, \mathbf{R})
$$

As this equation is a second-order differential equation in $3 N$ electron and $3 M$ nuclear (spatial) coordinates, several approximations are needed to solve it. In most cases, the starting point is the Born-Oppenheimer (BO) approximation.

The BO approximation involves neglecting the coupling between the motions of nuclei and electrons. As nuclei are much heavier than electrons, they can be regarded as stationary within the timescale of electronic motion. This means that their kinetic energies are negligible and that the potential due to internuclear interactions is just a constant. The electronic energy of the system at given nuclear configurations can therefore be computed by solving the electronic Schrödinger equation

$$
\hat{H}_{e} \Psi_{e}(\mathbf{r} ; \mathbf{R})=E_{e} \Psi_{e}(\mathbf{r} ; \mathbf{R})
$$

with

$$
\hat{H}_{e}=\hat{T}_{e}+\hat{V}_{e n}+\hat{V}_{e e}
$$

where $\hat{H}_{e}$ is the electronic Hamiltonian and $E_{e}$ is the associated electronic energy of the system. $\Psi_{e}(\mathbf{r} ; \mathbf{R})$ is the electronic wavefunction that depends explicitly on the electronic coordinates but only parametrically on the nuclear coordinates.

Returning to the molecular wavefunction $\Psi(\mathbf{r}, \mathbf{R})$, it can be expanded in terms of a complete orthonormal set of electronic wavefunctions $\left\{\Psi_{k e}(\mathbf{r} ; \mathbf{R}) \mid k=1,2, \ldots\right.$, $\infty\}$ with the nuclear wavefunctions $\varphi_{k}(\mathbf{R})$ as the expansion coefficients, 


$$
\Psi(\mathbf{r}, \mathbf{R})=\sum_{k=1}^{\infty} \varphi_{k}(\mathbf{R}) \Psi_{k e}(\mathbf{r} ; \mathbf{R})
$$

Substituting Eq. (2.8) in Eq. (2.5) and projecting the resulting equation onto the electronic wavefunction of a state $m$ followed by integration over the electronic coordinates yields

$$
\begin{aligned}
& {\left[E_{m e}+\hat{V}_{n n}+\hat{T}_{n}-E\right] \varphi_{k}(\mathbf{R})} \\
& -\sum_{A=1}^{M} \frac{1}{M_{A}} \sum_{k=1}^{\infty}\left\langle\Psi_{m e}\left|\nabla_{A}\right| \Psi_{k e}\right\rangle \nabla_{A} \varphi_{k}(\mathbf{R}) \\
& -\sum_{A=1}^{M} \frac{1}{2 M_{A}} \sum_{k=1}^{\infty}\left\langle\Psi_{m e}\left|\nabla_{A}^{2}\right| \Psi_{k e}\right\rangle \varphi_{k}(\mathbf{R})=0 .
\end{aligned}
$$

In the so-called adiabatic approximation, the electronic wavefunction is confined to a single eigenstate. Hence, all the terms that represent coupling between the eigenstates $k$ and $m$ in Eq. (2.9) are neglected. This gives

$$
\left[\left(E_{m e}+\hat{V}_{n n}\right)+\hat{T}_{n}+\left\langle\Psi_{m e}\left|\hat{T}_{n}\right| \Psi_{m e}\right\rangle\right] \varphi_{m}(\mathbf{R})=E \varphi_{m}(\mathbf{R})
$$

In the BO approximation, the $\left\langle\Psi_{m e}\left|\hat{T}_{n}\right| \Psi_{m e}\right\rangle$ term in the above equation, known as the diagonal correction term, is neglected. This results in a nuclear Schrödinger equation whose solution gives the vibrational and rotational energy levels of the electronic state $m$,

$$
\left[\varepsilon_{m e}+\hat{T}_{n}\right] \varphi_{m}(\mathbf{R})=E \varphi_{m}(\mathbf{R})
$$

with

$$
\varepsilon_{m e}=E_{m e}+\hat{V}_{n n}
$$

where the energy $\varepsilon_{m e}$ represents the potential for the motion of the nuclei. Hence, the BO approximation leads to the concept of a potential energy surface (PES). Although both the BO and adiabatic approximations are in general very good approximations, they break down in situations where the electronic wavefunction changes rapidly with the nuclear coordinates. This is usually the case when two or more configurations or states are nearly degenerate, such as at conical intersections that frequently occur in photochemical reactions. $^{37-42}$ 


\section{Computational Methods}

Overall, the application of the BO approximation to the molecular Schrödinger equation (2.5) involves two steps. In the first step, the nuclear kinetic energy and the internuclear repulsion terms are neglected in the Hamiltonian, and the resulting equation is solved for the electronic energy. In the second step, using the obtained electronic energy and reintroducing the neglected terms, the Schrödinger equation for the motion of the nuclei is solved. The focus of the present thesis is the first of these steps, i.e., solving the electronic Schrödinger equation (2.6). For the sake of brevity of notation, the "e" subscripts and the dependence on the coordinates in Eq. (2.6) are omitted hereafter. Thus, Eq. (2.6) can be written as

$$
\hat{H} \Psi=E \Psi
$$

The exact solution of this equation is precluded by the presence of the interelectronic interaction terms in the Hamiltonian $\hat{H}$ (of course, this is not the case with oneelectron systems), which prohibit the separation of the $N$-electron wavefunction $\Psi$ into individual one-electron components. Therefore, further approximations are required to solve this equation.

\subsubsection{Hartree-Fock Theory}

Hartree-Fock (HF) theory replaces the true interelectronic interactions, which preclude the exact solution of the Schrödinger equation, with mean-field interactions. This means that each electron feels a smeared-out charge distribution of all other electrons in the system. The $N$-electron wavefunction of the system is written as a single Slater determinant composed of $N$ orthonormal one-electron spin orbitals $\phi_{i}\left(\mathbf{x}_{\mathrm{i}}\right)$, called molecular orbitals (MOs),

$$
\Psi_{\mathrm{HF}}\left(\mathbf{x}_{1}, \mathbf{x}_{2}, \ldots, \mathbf{x}_{N}\right)=\frac{1}{\sqrt{N !}}\left|\begin{array}{cccc}
\phi_{1}\left(\mathbf{x}_{1}\right) & \phi_{2}\left(\mathbf{x}_{1}\right) & \cdots & \phi_{N}\left(\mathbf{x}_{1}\right) \\
\phi_{1}\left(\mathbf{x}_{2}\right) & \phi_{2}\left(\mathbf{x}_{2}\right) & \cdots & \phi_{N}\left(\mathbf{x}_{2}\right) \\
\phi_{1}\left(\mathbf{x}_{3}\right) & \phi_{2}\left(\mathbf{x}_{3}\right) & \cdots & \phi_{N}\left(\mathbf{x}_{3}\right) \\
\vdots & \vdots & \ddots & \vdots \\
\phi_{1}\left(\mathbf{x}_{N}\right) & \phi_{2}\left(\mathbf{x}_{N}\right) & \cdots & \phi_{N}\left(\mathbf{x}_{N}\right)
\end{array}\right|,
$$

where the $\left\{\mathbf{x}_{\mathrm{i}}\right\}$ represent both the spatial $\left(\mathbf{r}_{i}\right)$ and spin $\left(\boldsymbol{\sigma}_{i}\right)$ coordinates of the electrons. By variationally optimizing the electronic energy for this single-determinantal form of 
the wavefunction under the constraint of orthonormalization of the one-electron MOs $\phi_{i}$, we obtain the HF equations

$$
\hat{f}_{i} \phi_{i}=\varepsilon_{i} \phi_{i}
$$

where $\varepsilon_{i}$ is the energy of the MO $\phi_{i}$ and $\hat{f}_{i}$ is the one-electron Fock operator defined as

$$
\hat{f}_{i}=\hat{h}_{i}+\hat{v}_{H F}
$$

with

$$
\hat{h}_{i}=-\frac{1}{2} \nabla_{i}^{2}-\sum_{A=1}^{M} \frac{Z_{A}}{\mathbf{r}_{i A}} \text { and } \hat{v}^{\mathrm{HF}}=\sum_{j}\left(\hat{J}_{j}-\hat{K}_{j}\right) .
$$

Here, $\hat{h}_{i}$ includes the kinetic energy of the electrons and potential due to electronnucleus interactions. $\hat{v}^{\mathrm{HF}}$ is known as the HF potential that describes the average potential experienced by the $i^{\text {th }}$ electron due to electrostatic interactions $\left(\hat{J}_{j}\right)$ with all the remaining electrons and non-local exchange interactions $\left(\hat{K}_{j}\right)$ with electrons of like spin (also known as Fermi correlation).

In practice, the HF equations are solved by expanding the MOs as a linear combination of atomic orbitals (LCAO-MO), which are represented by a set of oneelectron functions $\left\{\mu_{k}\right\}$ known as basis functions (or basis set),

$$
\phi_{i}=\sum_{k} c_{k i} \mu_{k}
$$

to obtain matrix eigenvalue equations known as the Roothaan-Hall equations, ${ }^{43,44}$

$$
\mathbf{F C}=\mathbf{S C} \boldsymbol{\varepsilon}
$$

where $\mathbf{F}$ is the Fock matrix, $F_{k l}=\left\langle\mu_{k}|\mathbf{F}| \mu_{l}\right\rangle ; \mathbf{S}$ is the overlap matrix, $S_{k l}=\left\langle\mu_{k} \mid \mu_{l}\right\rangle ; \mathbf{C}$ is the matrix of MO coefficients, $\left\{\mathrm{c}_{k i}\right\}$; and $\varepsilon$ is the diagonal matrix of MO energies, $\left\{\varepsilon_{i}\right\}$. As the Fock matrix depends on the MO coefficients that are unknown, these equations are solved iteratively in a self-consistent way starting from an initial guess set of MO coefficients. The HF energy of the ground state is then calculated as

$$
E_{H F}=\sum_{i=1}^{N} \varepsilon_{i}-\sum_{i<j}\langle i j \| i j\rangle+V_{n n}
$$


The second term of this equation removes the error associated with double counting of the Coulomb and exchange potentials that arises from the summation of MO energies (in the first term).

A HF calculation is an example of an ab initio calculation, which means that it makes no approximation to the Hamiltonian or interelectronic interactions through empirical parameterization.

\subsubsection{Electron Correlation}

The basic idea of HF theory that electrons interact in a mean-field fashion is of course not entirely realistic. In reality, electrons tend to avoid each other more strongly and hence reside further apart than is predicted by HF theory. The difference between the "exact" energy $\left(E_{0}\right)$ and the HF energy $\left(E_{\mathrm{HF}}\right)$ at a given basis-set level is defined as the correlation energy $\left(E_{\text {corr }}\right),{ }^{45}$

$$
E_{\text {corr }}=E_{0}-E_{\mathrm{HF}} \text {. }
$$

Correlation effects can broadly be classified as static and dynamic effects. Static correlation is a long-range effect that comes into play when two or more configurations or states approach near degeneracy, such as in cleavage or formation of chemical bonds. In such cases, a single HF determinant is no longer appropriate for a proper description of the system under consideration and one needs to consider a linear combination of several Slater determinants. Static correlation effects are commonly treated using multi-configurational quantum chemical methods such as the complete active space self-consistent field (CASSCF) method. ${ }^{46}$

Dynamic correlation, on the other hand, is a short-range phenomenon that arises from the interelectronic repulsion terms in the Hamiltonian (called Coulomb correlation). In order to treat dynamic correlation, several different methods that start from a HF reference wavefunction have been developed. These methods are commonly known as post-HF methods. Examples of such methods are configuration interaction (CI), many-body perturbation theory (MBPT) and coupled-cluster (CC) approaches. The basic principles of these methods are briefly summarized below. For comprehensive accounts of these approaches, see e.g., Refs. [47-49]. 


\section{Methods}

In CI methods, the wavefunction is expressed as a linear combination of the HF determinant and several excited Slater determinants, obtained by replacing occupied MOs in the HF reference determinant with virtual MOs,

$$
\Psi_{\mathrm{CI}}=a_{0} \Psi_{\mathrm{HF}}+\sum_{\mathrm{S}} a_{1} \Psi_{\mathrm{S}}+\sum_{\mathrm{D}} a_{2} \Psi_{\mathrm{D}}+\sum_{\mathrm{T}} a_{3} \Psi_{\mathrm{T}}+\cdots
$$

where the subscripts $\mathrm{S}, \mathrm{D}$ and $\mathrm{T}$ refer to singly, doubly and triply excited determinants, respectively, formed by replacing one, two and three occupied MOs with virtual MOs. The expansion coefficients are determined by variationally optimizing the energy under the constraint of orthonormalization of the CI wavefunction. The variational problem is usually solved by recasting it into a matrix eigenvalue problem.

If one includes all possible excited determinants for a given basis set in the CI expansion, then the procedure is known as full CI. A full CI calculation with an infinite basis set would correspond to the "exact" (non-relativistic) solution of the Schrödinger equation. This implies that in order to get a good estimate of the "exact" solution, full CI calculations should be carried out with large basis sets, which is practical only for very small molecular systems. Therefore, CI expansions are often truncated by considering only a few types of determinants, for example, only singly and doubly excited ones (CISD). However, truncated CI methods lack sizeextensivity, which means that the portion of correlation energy captured by these methods decrease with increasing number of electrons in the system.

\section{MBPT Methods}

The basic idea of MBPT approaches is to partition the total Hamiltonian $\hat{H}$ of the system into two parts. A major part $\hat{H}^{0}$, for which exact eigenvalues and eigenfunctions are known and a minor unknown part $\hat{V}$ regarded as a perturbation to the major one,

$$
\hat{H}=\hat{H}^{0}+\lambda \hat{V},
$$




\section{Computational Methods}

where $\lambda$ is a dimensionless arbitrary parameter representing the strength of the perturbation. The wavefunction and energy of the perturbed system are expanded in power series of $\lambda$ as

$$
\Psi=\Psi^{(0)}+\lambda^{1} \Psi^{(1)}+\lambda^{2} \Psi^{(2)}+\lambda^{3} \Psi^{(3)}+\cdots
$$

and

$$
E=E^{(0)}+\lambda^{1} E^{(1)}+\lambda^{2} E^{(2)}+\lambda^{3} E^{(3)}+\cdots,
$$

where the $\Psi^{(0)}$ and $\mathrm{E}^{(0)}$ correspond to the unperturbed system and the superscripts 1, 2, $3, \ldots$, correspond to the first, second, third-order corrections, and so on.

Møller and Plesset (MP) considered the zeroth order Hamiltonian $\hat{H}^{0}$ to be the sum of the one-electron Fock operators $\hat{f}_{i}$,

$$
\hat{H}^{0}=\sum_{i=1}^{N} \hat{f}_{i}
$$

Then, by substituting Eqs. (2.23)-(2.26) in the electronic Schrödinger equation and solving the equations that are linear and quadratic in $\lambda$, they obtained the first (MP1) and second-order (MP2) corrections to the energy and the first-order correction to the wavefunction as

$$
\begin{gathered}
E^{(1)}=\left\langle\Psi^{(0)}|\hat{V}| \Psi^{(0)}\right\rangle=-\sum_{i<j}\langle i j \| i j\rangle, \\
E^{(2)}=\left\langle\Psi^{(0)}|\hat{V}| \Psi^{(1)}\right\rangle=\sum_{i<j} \sum_{a<b} \frac{|\langle a b \| i j\rangle|^{2}}{\varepsilon_{i}+\varepsilon_{j}-\varepsilon_{a}-\varepsilon_{b}}, \\
\left|\Psi^{(1)}\right\rangle=\sum_{i<j} \sum_{a<b} \frac{\langle a b \| i j\rangle}{\varepsilon_{i}+\varepsilon_{j}-\varepsilon_{a}-\varepsilon_{b}}\left|\Psi^{(0)}\right\rangle .
\end{gathered}
$$

Here, the indices $i$ and $j$ correspond to occupied orbitals, and the indices $a$ and $b$ correspond to virtual orbitals. The first-order correction is nothing but the correction for double counting of the Coulomb and exchange potentials that arises from the summation of MO energies, and is already present in the HF energy given by Eq. (2.20). Therefore, the second-order energy correction is the first step beyond the HF energy. Although it is possible to further improve up on this energy by going to 
higher orders of perturbation (MP3, MP4, MP5, ...), a convergent behavior is not guaranteed by these approaches.

\section{Methods}

$\mathrm{CC}$ methods are the most accurate post-HF methods available for treating dynamical correlation effects in a systematic and hierarchical manner. These methods employ an exponential parameterization of the reference $\mathrm{HF}$ wavefunction,

$$
\left|\Psi_{\mathrm{CC}}\right\rangle=e^{\hat{T}}\left|\Psi_{\mathrm{HF}}\right\rangle
$$

where $\hat{T}$ is the cluster operator defined as the sum of $N$ excitation operators for a system with $N$ electrons,

$$
\hat{T}=\hat{T}_{1}+\hat{T}_{2}+\hat{T}_{3}+\cdots+\hat{T}_{N},
$$

where the subscripts $1,2,3, \ldots, N$, correspond to number of simultaneous electron excitations generated by the corresponding operator. For example,

$$
\begin{gathered}
\hat{T}_{1}\left|\Psi_{H F}\right\rangle=\sum_{i}^{\mathrm{occ}} \sum_{a}^{\mathrm{virt}} t_{i}^{a}\left|\Psi_{i}^{a}\right\rangle \\
\hat{T}_{2}\left|\Psi_{H F}\right\rangle=\sum_{j>i}^{\mathrm{occ}} \sum_{b>a}^{\mathrm{virt}} t_{i j}^{a b}\left|\Psi_{i j}^{a b}\right\rangle
\end{gathered}
$$

represent the action of single and double excitation operators on the HF reference wavefunction, with associated cluster amplitudes $t_{i}^{a}$ and $t_{i j}^{a b}$. Substituting the exponential ansatz (2.30) in the electronic Schrödinger equation gives

$$
\hat{H} e^{\hat{T}}\left|\Psi_{\mathrm{HF}}\right\rangle=E e^{\hat{T}}\left|\Psi_{\mathrm{HF}}\right\rangle .
$$

Projecting this equation onto the HF reference wavefunction yields

$$
E_{\mathrm{CC}}=\left\langle\Psi_{\mathrm{HF}}|\hat{H}| e^{\hat{T}} \Psi_{\mathrm{HF}}\right\rangle
$$

as the CC energy expression. Further, the expression for cluster amplitudes can be obtained by projecting Eq. (2.34) onto the space of all excited determinants produced 


\section{Computational Methods}

by the cluster operator $\hat{T}$. The resulting equations are then solved in an iterative way to obtain the cluster amplitudes.

With the inclusion of all the $N$ excitation operators in the cluster operator, this approach would be equivalent to doing a full CI, which is not practical for all but very small systems. In practice, the cluster expansion is truncated after a few terms, giving a hierarchy of CC approaches such as CCD, CCSD and CCSDT corresponding to $\hat{T}=\hat{T}_{2}, \hat{T}=\hat{T}_{1}+\hat{T}_{2}$ and $\hat{T}=\hat{T}_{1}+\hat{T}_{2}+\hat{T}_{3}$, respectively. Unlike truncated-CI methods, truncated-CC methods are size-extensive as the exponential form of the wavefunction in these methods ensures the correct scaling behavior of energy.

Although wavefunction-based correlation methods offer a rigorous approach to treat electron correlation and to achieve systematic improvements upon the HF energy, these methods scale poorly with system size. A cost-effective computational approach to include dynamical correlation effects in large molecular systems is density functional theory.

\subsubsection{Density Functional Theory}

In the following discussion, the mathematical concept of a functional is frequently used. Loosely, while a function maps a number into a number, a functional maps a function into a number. The notation $\mathrm{F}[\mathrm{f}(\mathbf{r})]$ is used to indicate that $\mathrm{F}$ is a functional of the function $\mathrm{f}(\mathbf{r})$.

The central quantity of density functional theory (DFT) is the electron density of the system. Although the importance of the electron density as a fundamental quantity was recognized already in the 1920s, no rigorous mathematical framework using electron densities to describe chemical bonding emerged until the 1960s. In 1964, Hohenberg and Kohn (HK) formulated and proved two theorems, ${ }^{50}$ which form the foundation of modern DFT.

The first HK theorem states that for a system of interacting electrons in an external potential $\left(V_{e x t}\right)$, the ground-state electron density $\rho(\mathbf{r})$ uniquely (within a constant) determines $V_{\text {ext }}$. As $V_{\text {ext }}$ fixes the Hamiltonian, this means that the electron density uniquely determines the Hamiltonian. The electronic energy can therefore be expressed as

$$
E[\rho]=T_{e}[\rho]+E_{e e}[\rho]+V_{e x t}[\rho]=F[\rho]+V_{e x t}[\rho],
$$


where $F[\rho]$ contains the kinetic energy of electrons $T_{e}[\rho]$ and the interelectronic repulsion $E_{e e}[\rho]$. The functional forms of $T_{e}[\rho]$ and $E_{e e}[\rho]$ are independent of the system at hand. $F[\rho]$ is therefore a universal functional.

The second HK theorem states that the exact ground-state electronic energy can be obtained by variational minimization of the universal functional $F[\rho]$ over all possible electron densities. However, the exact functional forms of the kinetic energy and interelectronic repulsion terms that constitute $F[\rho]$ are unknown, which precludes direct access to the exact energy.

In 1965, Kohn and Sham came up with a proposal for calculating the major portion of the kinetic energy in an exact way. ${ }^{51}$ They proposed a fictitious system of $N$ non-interacting electrons moving in an effective potential $v_{s}(\mathbf{r})$ such that this system has the same electron density as the real interacting system. The exact wavefunction of the non-interacting system is a single Slater determinant composed of $\operatorname{MOs},\left\{\phi_{i}(\mathbf{r}) \mid\right.$ $i=1,2, \ldots, N\}$, which can be obtained by solving $N$ one-electron Schrödinger-like equations known as the Kohn-Sham (KS) equations

$$
\left[-\frac{1}{2} \nabla^{2}+v_{s}(\mathbf{r})\right] \phi_{i}(\mathbf{r})=\varepsilon_{i} \phi_{i}(\mathbf{r}) .
$$

The exact kinetic energy of the fictitious non-interacting system can then be calculated from the MOs as

$$
T_{s}[\rho]=-\frac{1}{2} \sum_{j=1}^{N}\left\langle\phi_{i}\left|\nabla^{2}\right| \phi_{i}\right\rangle .
$$

Returning to the real interacting system, the universal functional $F[\rho]$ can be written as

$$
F[\rho]=T_{\mathrm{s}}[\rho]+J[\rho]+E_{\mathrm{xc}}[\rho]
$$

where $J[\rho]$ is the classical Coulomb potential and $E_{\mathrm{xc}}[\rho]$ is the exchange-correlation energy defined as

$$
E_{\mathrm{xc}}[\rho]=\left(T[\rho]-T_{\mathrm{s}}[\rho]\right)+\left(E_{e e}[\rho]-J[\rho]\right) .
$$

The exchange-correlation energy contains two correction terms, one associated with replacing the kinetic energy of the real system with that of the fictitious one and the 
other related to the classical treatment of interelectronic interactions. The exact energy functional can now be expressed as

$$
\begin{gathered}
E[\rho]=T_{\mathrm{s}}[\rho]+J[\rho]+E_{\mathrm{xc}}[\rho]+V_{\mathrm{ext}}[\rho], \\
\text { or } \quad E[\rho]=T_{s}[\rho]+\frac{1}{2} \iint \frac{\rho(\mathbf{r}) \rho\left(\mathbf{r}^{\prime}\right)}{\left|\mathbf{r}-\mathbf{r}^{\prime}\right|} d \mathbf{r} d \mathbf{r}^{\prime}+E_{\mathrm{xc}}[\rho]+\int v_{\mathrm{ext}}(\mathbf{r}) \rho(\mathbf{r}) d \mathbf{r} .
\end{gathered}
$$

Minimizing the energy functional $E[\rho]$ for a given number of electrons yields

$$
\begin{aligned}
& \int \delta \rho(\mathbf{r}) d \mathbf{r}\left\{\frac{\delta T_{s}[\rho]}{\delta \rho(\mathbf{r})}+v_{\mathrm{s}}(\mathbf{r})-\varepsilon\right\}=0, \\
& v_{s}(\mathbf{r})=\int \frac{\rho\left(\mathbf{r}^{\prime}\right)}{\left|\mathbf{r}-\mathbf{r}^{\prime}\right|} d \mathbf{r}^{\prime}+v_{\mathrm{xc}}[\mathbf{r}]+v_{\mathrm{ext}}(\mathbf{r}),
\end{aligned}
$$

where $v_{\mathrm{xc}}(\mathbf{r})$ is known as the exchange-correlation potential, defined as

$$
v_{\mathrm{xc}}[\mathbf{r}]=\frac{\delta E_{\mathrm{xc}}[\rho]}{\delta \rho(\mathbf{r})} .
$$

The electron density can be obtained by first solving the KS equations (2.37) for the non-interacting system under the effective potential $v_{s}(\mathbf{r})$ given by Eq. (2.44), and then expressing the density as

$$
\rho(r)=\sum_{i=1}^{N}\left|\phi_{i}\right|^{2}
$$

As $v_{s}(\mathbf{r})$ depends on the electron density that is unknown, the KS equations must be solved iteratively from a trial electron density to construct an initial guess for $v_{\mathrm{s}}(\mathbf{r})$. The KS energy can then be determined as

$$
E_{\mathrm{KS}}=\sum_{i} \varepsilon_{i}-\frac{1}{2} \iint \frac{\rho(\mathbf{r}) \rho\left(\mathbf{r}^{\prime}\right)}{\left|\mathbf{r}-\mathbf{r}^{\prime}\right|} d \mathbf{r} d \mathbf{r}^{\prime}+E_{\mathrm{xc}}[\rho(\mathbf{r})]-\int v_{\mathrm{xc}}[\mathbf{r}] \rho(\mathbf{r}) d \mathbf{r}
$$

If the exact functional form of the exchange-correlation energy $E_{\mathrm{XC}}$ were known, then the expression (2.47) would yield the "exact" energy. However, its explicit form is unknown, and developing better and better approximations to this functional is one of the central themes of modern DFT research. 


\section{Exchange-Correlation Functionals}

The construction of exchange-correlation functionals broadly follows two different approaches. In the first approach, the functionals are built to satisfy exact constraints derived from theoretical arguments. The second approach is based on parameterizing the functionals by fitting them to experimental data or high-level wavefunction-based correlation treatments. Functionals constructed using fitting approaches often lack transferability compared to those derived from exact constraints alone. ${ }^{52}$

Alternatively, based on the ingredients present, the exchange-correlation functionals are often associated with different rungs of "Jacob's ladder". 53 Climbing up the ladder involves increasing the number and nature (local or non-local) of ingredients. The functionals at the first or lowest rung employ a uniform electron gas model to calculate the exchange-correlation energy. The electron density $\rho$, which is assumed to be constant throughout the system, is the only ingredient of these functionals. This approximation, known as the local density approximation (LDA), is particularly useful for systems with slowly varying electron densities but is far from practically useful for molecules. At the second rung, the functionals include dependence on the $\rho$ as well as its gradient $\nabla \rho$. This is referred to as generalized gradient approximation (GGA). Examples of such functionals are BLYP $^{54,55}$ and BP86. ${ }^{54,56}$ Moving to the third rung of functionals, the so-called meta-GGAs incorporate the Laplacian of the electron density $\nabla^{2} \rho$ (and also the kinetic energy density) in addition to $\rho$ and $\nabla \rho$.

At the fourth rung, the functionals contain exact HF exchange, along with $\rho$ and $\nabla \rho$. These functionals can further be classified as global or range-separated hybrids depending on whether they contain a fixed or varying (with the interelectronic distance) amount of HF exchange. The B3LYP ${ }^{55,57,58}$ global hybrid contains $20 \%$ exact exchange and is one of the most widely used methods in contemporary quantum chemistry. The fifth rung of functionals involves dependence on virtual KS orbitals. Examples of these functionals are double-hybrids such as the B2-PLYP functional, ${ }^{59}$ which in addition to the HF exchange also include a second-order perturbative correlation term. 


\subsection{Modeling Excited Electronic States}

An excited electronic state is a state with higher electronic energy than the ground state at a given molecular geometry. For example, such a state can be formed by absorption of UV or visible light, but can also decay back to the ground state by means of thermal, photophysical or photochemical channels. These decay channels often involve avoided or surface crossings with other electronic states, which makes it more challenging to describe excited states than ground states. The simplest approach to treat excited states is the so-called $\triangle \mathrm{SCF}$ approach, which is useful when the excited state of interest has a different spatial or spin symmetry than the ground state. More commonly used methods for modeling excited states fall into two categories: single-configurational and multi-configurational methods. The former approaches are mainly capable of treating excited states formed by single electron excitations from the ground state (known as single-reference excited states), whereas the latter are more general in their applicability.

\subsubsection{Single-Configurational Methods}

Single-configurational methods for modeling excited states are essentially of two types. ${ }^{60}$ The first type of methods explicitly computes wavefunctions and energies of all individual states of interest to obtain associated excitation energies. CI methods are of this type. The second type of methods starts from a ground-state reference wavefunction and treats electronic excitations as perturbations of the wavefunction due to interactions with an external field. The excitation energies are computed without any explicit calculations on individual excited states. The quality of these approaches relies on the quality of ground-state reference wavefunction. Equation-ofmotion CC (EOM-CC), ${ }^{61,62}$ linear response CCn approaches ${ }^{63}$ and time-dependent density functional theory (TD-DFT) ${ }^{64-70}$ methods belong to this category. Below, these two types of methods are briefly summarized.

\section{Methods}

CI singles (CIS $)^{60}$ is the simplest wavefunction-based approach for modeling singlereference excited states. In CIS, the electronic wavefunction is expressed as a linear 
combination of all possible singly excited Slater determinants, obtained by replacing an occupied MO with a virtual MO in the HF reference determinant,

$$
\Psi_{\mathrm{CIS}}=\sum_{i}^{\text {occupied virtual }} \sum_{a} c_{i a} \Psi_{i}^{a}
$$

The excitation energies and expansion coefficients (known as CI coefficients) are obtained by diagonalizing the CIS matrix formed from the HF reference and all singly excited determinants. As the HF reference state does not couple with singly excited states (Brillouin theorem), the diagonalization is only needed in the space of excited determinants. The main drawback of the CIS method is the absence of electron correlation effects. A possible way to incorporate correlation effects is by including double excitations perturbatively with the CIS(D) method. ${ }^{71}$

\section{Methods}

$\mathrm{CC}$ methods are one of the most accurate wavefunction-based approaches available for treating single-reference excited states in small- and medium-sized molecules. Examples of commonly used CC formulations for modeling excited states are EOM$\mathrm{CC}$ and the linear response CCn approaches. The basic idea of these approaches, referred to as propagator approaches, ${ }^{72}$ is that the physical property of interest is calculated by means of a propagator associated with it. Electronic excitations are modeled by considering the ground-state dynamic polarizability $\alpha(\omega)$ propagator. The excitation energies and transition dipole moments are obtained without having to compute wavefunctions of individual excited states, from the expression for $\alpha(\omega)$,

$$
\alpha(\omega)=\sum_{n>0} \frac{2 \omega_{n o}\left|\left\langle\Psi_{n}|\mu| \Psi_{0}\right\rangle\right|^{2}}{\omega_{n 0}^{2}-\omega^{2}} .
$$

This expression diverges at $\omega_{n 0}=\omega$, where $\omega_{n 0}$ corresponds to the frequency of absorption between the states 0 (ground state) and $n$ (excited state). The excitation energies can then be obtained as $E_{n}-E_{0}=\hbar \omega_{n 0}$, and the associated oscillator strengths can be calculated from the transition dipole moments $\left\langle\Psi_{n}|\mu| \Psi_{0}\right\rangle$ as

$$
f_{n 0}=\frac{2}{3} \omega_{n o}\left|\left\langle\Psi_{n}|\mu| \Psi_{0}\right\rangle\right|^{2} .
$$




\section{Computational Methods}

One of the most cost-effective CC schemes available today for modeling single-reference excited states is the approximate coupled-cluster singles and doubles (CC2) method. ${ }^{63}$

\section{TD-DFT}

TD-DFT is an extension of conventional ground-state DFT to treat excited electronic states. The time-dependent analogs of the HK theorems, known as the Runge-Gross (RG) theorems, ${ }^{64}$ form the basis of TD-DFT. The time-dependent KS equations take the form

$$
\left[-\frac{1}{2} \nabla^{2}+v_{s}(\mathbf{r}, t)\right] \phi_{i}(\mathbf{r}, t)=i \hbar \frac{\partial \phi_{i}(\mathbf{r}, t)}{\partial t},
$$

with

$$
v_{s}(\mathbf{r}, t)=v_{\mathrm{ext}}(\mathbf{r}, t)+\int \frac{\rho\left(\mathbf{r}^{\prime}, t\right)}{\left|\mathbf{r}-\mathbf{r}^{\prime}\right|} d \mathbf{r}^{\prime}+v_{\mathrm{xc}}(\mathbf{r}, t) .
$$

In the adiabatic approximation, it is assumed that the exchange-correlation potential at any time $t$ depends only on the density at that particular time $\rho_{\mathrm{t}}$,

$$
v_{\mathrm{xc}}[\rho](\mathbf{r}, t) \approx \frac{\delta E_{\mathrm{xc}}\left[\rho_{t}\right](\mathbf{r})}{\delta \rho_{t}(\mathbf{r})}=v_{\mathrm{xc}}\left[\rho_{t}\right](\mathbf{r}) .
$$

This approximation enables the use of ground-state exchange-correlation potentials for excited-state calculations. The derivative of the exchange-correlation potential with respect to the density within the adiabatic approximation is given by

$$
f_{\mathrm{xc}}[\rho]\left(\mathbf{r}, \mathbf{r}^{\prime}\right)=\frac{\delta v_{\mathrm{xc}}\left[\rho_{t}\right](\mathbf{r})}{\delta \rho_{t}\left(\mathbf{r}^{\prime}\right)}=\frac{\delta^{2} E_{\mathrm{xc}}\left[\rho_{t}\right](\mathbf{r})}{\delta \rho_{t}\left(\mathbf{r}^{\prime}\right) \delta \rho_{t}(\mathbf{r})}
$$

where $f_{\mathrm{xc}}$ is known as the exchange-correlation kernel and is used to find the linear response of the electron density to a time-dependent external field that is considered as a slowly acting perturbation on the electron density. The adiabatic approximation usually yields good results for excitations to low-lying states. ${ }^{67}$

In the commonly used linear response formalism of TD-DFT (LR-TD-DFT), the excitation energies are obtained as solutions of the pseudo-eigenvalue problem 


$$
\left[\begin{array}{ll}
\mathbf{A} & \mathbf{B} \\
\mathbf{B}^{*} & \mathbf{A}^{*}
\end{array}\right]\left[\begin{array}{l}
\mathbf{X}_{I} \\
\mathbf{Y}_{I}
\end{array}\right]=\omega_{I}\left[\begin{array}{cc}
1 & 0 \\
0 & -1
\end{array}\right]\left[\begin{array}{l}
\mathbf{X}_{I} \\
\mathbf{Y}_{I}
\end{array}\right],
$$

where the matrix elements of $\mathbf{A}$ and $\mathbf{B}$ are given by

$$
\begin{aligned}
& A_{i a, j b}=\delta_{i j} \delta_{a b}\left(\varepsilon_{a}-\varepsilon_{i}\right)+(i a \mid j b)+\left(i a\left|f_{x c}\right| j b\right), \\
& B_{i a, j b}=(i a \mid j b)+\left(i a\left|f_{x c}\right| j b\right) .
\end{aligned}
$$

The eigenvalues $\omega_{I}$ provide access to the excitation energies and the eigenvectors $\mathbf{X}_{I}$ and $\mathbf{Y}_{I}$ yield the oscillator strengths.

Although TD-DFT works best for low-lying valence excited states, recently developed range-separated hybrid functionals like CAM-B3LYP ${ }^{73}$ and $\omega B$ 97X-D ${ }^{74}$ enable also the description of charge-transfer states. Thanks to the efficient implementations of analytic energy gradients for excited states, ${ }^{75-79}$ TD-DFT has nowadays become a powerful tool for modeling equilibrium geometries of excited states in large molecular systems. However, modeling the full course of photochemical reactions is still problematic as this typically involves describing neardegeneracy effects, which cannot be accounted for with conventional TD-DFT due to its single-reference nature. A possible solution to this problem is to employ the spinflip TD-DFT method, ${ }^{80}$ which incorporates some double excitation character.

\section{Composite Procedures}

Composite procedures are multi-step quantum chemical approaches that combine calculations from different levels of theory to obtain accurate results at moderate computational costs. Examples of such procedures are Gaussian- $n(\mathrm{G} n)^{81-84}$ and Weizmann- $n(\mathrm{~W} n)^{85-87}$ approaches that combine a high-level correlation method and a small basis set with a low-level correlation method and a larger basis set. These procedures are routinely used in studies of ground electronic states to obtain accurate thermochemical data. However, the potential of composite procedures to model excited electronic states is not as well explored as their utility to treat ground states. However, some notable contributions to this field have been made by Grimme ${ }^{88}$ and Jacquemin $^{89-92}$ and their coworkers, who aimed to simplify the calculation of $0-0$ excitation energies $\left(\Delta E_{00}\right.$, i.e., energy differences between ground and excited states in their lowest vibrational levels) using composite procedures. These procedures 
combined geometries and zero-point vibrational energy (ZPVE) corrections obtained from TD-DFT calculations with transition energies from wavefunction-based methods such as CIS(D) and CC2.

In this thesis, a novel composite procedure that combines TD-DFT and CC2 calculations to obtain CC2-quality $\Delta E_{00}$ energies is proposed. In this procedure, excited-state relaxation energies $\left(\Delta \Delta E_{\text {ad }}\right)$ and differences in ZPVE corrections between ground and excited states $\left(\Delta \Delta E_{00}\right)$ from TD-DFT calculations are combined with vertical excitation energies $\left(\Delta E_{\mathrm{ve}}\right.$, i.e., energy differences between ground and excited states at the ground-state equilibrium geometry) from $\mathrm{CC} 2$ calculations, as shown in Figure 2.1.

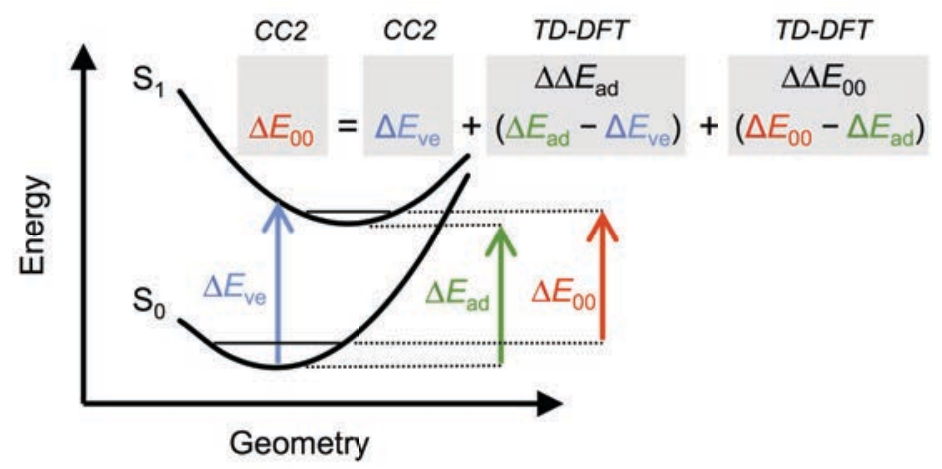

Figure 2.1 Different types of excitation energies and definition of a composite procedure to calculate $\Delta E_{00}$ energies.

In Figure 2.1, $\Delta E_{\mathrm{ad}}$ is the adiabatic excitation energy (i.e., the energy difference between ground and excited states at their respective equilibrium geometries).

\subsubsection{Multi-Configurational Methods}

Single-configurational methods like CC2 and TD-DFT are particularly well-suited for modeling absorption properties and equilibrium excited-state geometries but are usually inadequate for a rigorous description of the full mechanisms of photochemical reactions. This is because such reactions are typically mediated by non-radiative decay channels, called conical intersections, ${ }^{37-42}$ at which two states become 
degenerate. To describe these situations, methods that invoke multi-determinantal description of the states, such as CASSCF, are essential.

\section{CASSCF}

The first step of a CASSCF calculation is to select a subset of MOs based on their perceived importance for describing the state of interest. These orbitals constitute the so-called active space and all the remaining orbitals form the inactive space. A full CI calculation is then performed within the active space by variationally optimizing both the $\mathrm{CI}$ and MO coefficients, in contrast to $\mathrm{CI}$ calculations that optimize only the CI coefficients. The variational process also optimizes the inactive MOs but only at the HF level.

Application of the CASSCF method for studying excited states is often plagued with a problem termed "root flipping". This occurs if the excited state of interest has a significantly different charge distribution from that of a lower lying state $j$ of the same symmetry, which implies that the orbitals optimized for the excited state are very poor for the description of the state $j$. This leads to rise in the energy of the state $j$ and can result in reordering of the energies of the two states. A useful solution to this problem is to employ a state-averaged CASSCF (SA-CASSCF) procedure, ${ }^{93}$ which involves optimizing the orbitals for the average energy of several states,

$$
E_{\text {aver }}=\sum_{n} \omega_{n} E_{n},
$$

where $\omega_{n}$ is the weight of state $n$. With equal weights for all the considered states, the resulting orbitals of such optimization procedure describe all the states equally well (but are not optimal for any of the states). Another advantage of SA-CASSCF calculations is that all calculated states are orthogonal to each other, which is not the case with state-specific CASSCF calculations.

One limitation of the CASSCF method is that it misses the short-range dynamic correlation effects that are different for different excited states and therefore are important to obtain a good quantitative description of the states. Typically, these effects are accounted for by means of second-order perturbation theory calculations using a CASSCF reference wavefunction, referred to as the CASPT2 method. ${ }^{94,95}$ 


\section{Computational Methods}

\section{CASPT2}

The starting point of a CASPT2 calculation is the CASSCF reference wavefunction $\mid 0>$ computed for the state of interest. The first order correction to this wavefunction includes all possible singly and doubly excited configurations $\{|j\rangle, j=1,2, \ldots, M\}$ with respect to the reference $|0\rangle$,

$$
\left|\Psi^{(1)}\right\rangle=\sum_{j=1}^{M} C_{j}|j\rangle
$$

with

$$
|j\rangle=\hat{E}_{p q} \hat{E}_{r s}|0\rangle
$$

where the operators $\hat{E}_{p q}$ and $\hat{E}_{r s}$ are excitation operators. The coefficients $C_{j}$ are determined by solving the system of linear equations

$$
\sum_{j=1}^{M} C_{j}\left\langle i\left|\hat{H}^{0}-E^{(0)}\right| j\right\rangle=-\langle i|\hat{H}| 0\rangle, \quad i=1,2, \ldots, M
$$

where $E^{(0)}=\left\langle 0\left|\hat{H}^{0}\right| 0\right\rangle$ is the zeroth-order energy. The zeroth order Hamiltonian $\hat{H}^{0}$ is defined with the prerequisite that it must be equivalent to the MP Hamiltonian for a closed-shell HF reference wavefunction. Typically, $\hat{H}^{0}$ is expressed in terms of a generalized Fock operator $(\hat{F})$, which is written as a sum of diagonal $\left(\hat{F}_{D}\right)$ and nondiagonal contributions $\left(\hat{F}_{N}\right)$,

$$
\hat{F}=\hat{F}_{D}+\hat{F}_{N} .
$$

In the simplest case of $\hat{F}=\hat{F}_{D}$, Eq. (2.60) can be expressed as

$$
\sum_{j=1}^{M} C_{j}\left\langle i\left|\hat{F}_{D}\right| j\right\rangle-E^{(0)} \sum_{j=1}^{M} C_{j}\langle i \mid j\rangle=-\langle i|\hat{H}| 0\rangle,
$$

which can be written in matrix form as

$$
\left[\mathbf{F}_{D}-E^{(0)} \mathbf{S}\right] \mathbf{C}=-\mathbf{V}
$$

The second-order correction to the energy is given by the matrix product $\mathbf{V}^{\dagger} \mathbf{C}$, where $\mathbf{V}$ contains the elements corresponding to interaction between the excited 
configurations $\mid i>$ and the CASSCF reference wavefunction $\mid 0>$, and $\mathbf{C}$ is the vector of the coefficients $C_{j}$.

\subsection{Non-Adiabatic Molecular Dynamics Simulations}

The excited-state methods described in the previous section can in principle be employed to generate a static BO PES of a photoinduced excited state through the calculation of electronic excitation energies at different nuclear geometries. With an appropriate choice of method and reaction coordinate, such a PES can provide useful mechanistic insights into a photochemical reaction occurring in the corresponding state. However, this static mechanistic description has limitations. First, as excitedstate PESs are often much flatter than those of ground states, it is difficult to obtain quantitative information about the timescales of photochemical reactions from static calculations. Moreover, static calculations provide very little information about the efficiencies of these reactions. Second, photochemical reactions often involve two or more BO PESs that are non-adiabatically coupled, which means that modeling a single BO PES is insufficient for a rigorous description of the reaction mechanism. Therefore, one needs to consider the dynamics of the nuclei (and possibly also the electrons) and allow for population transfer between coupled BO PESs to obtain more comprehensive insights into these reactions. Such dynamics are known as nonadiabatic molecular dynamics (NAMD). ${ }^{96-105}$

A common approach for carrying out NAMD simulations is to propagate the nuclei classically on a single BO PES using forces obtained "on the fly" from electronic structure calculations but with the allowance for hopping to a different BO PES in the regions of considerable non-adiabatic coupling and small energy gap between the PESs. The probability of hopping is computed by means of trajectory surface-hopping (TSH) algorithms such as Tully's fewest-switches algorithm (FSA) ${ }^{96}$ or approximate switching algorithms ${ }^{100-102}$ such as Robb's diabatic hopping algorithm (DHA). ${ }^{100,101}$ In Tully's FSA, the hopping probability is proportional to the variation in the populations of different electronic states as well as to the non-adiabatic coupling between the states. Obtaining the state populations requires solving the timedependent Schrödinger equation for electrons at every time step along the trajectory, which limits the practical applicability of this approach. 
In Robb's DHA, the hopping probability between the electronic states $\Psi_{1}$ and $\Psi_{2}$ are computed by means of Landau-Zener formula as

$$
\begin{gathered}
P=\exp \left[-\frac{\pi}{4} \frac{\Delta E}{h D}\right], \\
D=\left\langle\Psi_{1} \mid \frac{\partial \Psi_{2}}{\partial t}\right\rangle .
\end{gathered}
$$

Here, $\Delta E$ is the energy difference between the states and $D$ arises from the nonadiabatic coupling $\left\langle\Psi_{1} \mid \nabla_{\mathbf{R}} \Psi_{2}\right\rangle$ between the states, with $\mathbf{R}$ representing the nuclear coordinates. For a small time step $\Delta t$ for the nuclear motion, $D$ is numerically approximated as

$$
\left\langle\Psi_{1} \mid \frac{\partial \Psi_{2}}{\partial t}\right\rangle=\frac{\left\langle\Psi_{1}(t) \mid \Psi_{2}(t+\Delta t)\right\rangle}{\Delta t} .
$$

As the hopping probability is computed without the need to solve the time-dependent electronic Schrödinger equation, this algorithm is practical for treating large molecular systems.

\subsection{Solvent Effects}

The kinetics and thermodynamics of chemical reactions are determined not only by intrinsic molecular properties but also by the reaction medium. Typically, synthetic chemical reactions are carried out in solution. Therefore, modeling solvent effects are essential for appropriate comparison of computational and experimental data. Methods for modeling solvent effects can broadly be classified into two types: explicit and implicit solvation models. The former methods explicitly describe the individual solvent molecules and therefore include specific solute-solvent interactions. Examples of such methods are so-called QM/MM approaches, ${ }^{106-108}$ in which the solute is treated quantum mechanically and the solvent is treated classically using molecular mechanics methods. In implicit solvation methods, the solvent is treated as a polarizable continuum characterized by its dielectric constant. These methods, commonly known as polarizable continuum models, ${ }^{109-112}$ are briefly described below. 


\subsubsection{Polarizable Continuum Models}

In a polarizable continuum model (PCM), the solvent is treated as a uniform dielectric continuum, and the solute is embedded into a cavity in the solvent formed by a set of interlocking atomic spheres. The interface between the solute and solvent defines the cavity surface. The solute charge distribution polarizes the cavity surface, which, in turn, perturbs the solute charge density. The interactions between the solute and solvent are considered to be a perturbation $\hat{V}$ on the gas-phase Hamiltonian $\hat{H}^{(0)}$ of the solute

$$
\left[\hat{H}^{(0)}+\hat{V}\right] \Psi=E \Psi
$$

where $\Psi$ is the electronic wavefunction of the polarized solute in solution and $E$ is the associated energy. The interaction energy, known as free energy of solvation, has both electrostatic and non-electrostatic contributions.

$$
\Delta G_{\text {solv }}=\Delta G_{\text {electrostatic }}+\Delta G_{\text {non-electrostatic }} .
$$

In the commonly used SMD model ${ }^{112}$ for computing free energies of solvation, the electrostatic free-energy contributions arising from solute-solvent mutual polarization effects are computed by first partitioning the cavity surface into small discrete elements of apparent surface charges $q_{i}$ with positions $r_{i}$, and then solving the non-homogeneous Poisson's equation for electrostatics. The nonelectrostatic free-energy contributions arising from cavitation, dispersion and solvent structural changes are computed from empirical surface tensions of all the individual atoms of the solute. 
Computational Methods 


\section{Light-Driven Rotary Molecular Motors}

Light-driven rotary molecular motors are molecules that produce repetitive $360^{\circ}$ unidirectional rotary motion around a carbon-carbon ${ }^{6-10,17-31,113,114}$ or a carbonnitrogen $^{115,116}$ double bond by absorbing UV or visible light. In this thesis, two different classes of molecular motors are studied: sterically overcrowded alkenes (Papers I-IV) and protonated Schiff bases (Paper V). Both these classes of motors produce rotary motion around a carbon-carbon double bond.

\subsection{Sterically Overcrowded Alkenes}

A $360^{\circ}$ rotation of an overcrowded alkene-based molecular motor involves four discrete steps of which two are activated by light and the other two by heat. The overall rotary cycle is shown in Figure 3.1 for the case of molecular motor $\mathbf{1}^{6}$ (shown in the Introduction), and can be described as follows. In the first step, irradiation of the $(P, P)$-trans-1 isomer with UV light induces a trans $\rightarrow$ cis photoisomerization about the central double bond to yield the $(M, M)$-cis-2 isomer. This reaction inverts the helicities of the two motor halves from $P$ to $M$ and changes the orientations of the two methyl substituents from a favorable axial in $(P, P)$-trans-1 to a strained equatorial in $(M, M)$-cis-2.

In $(M, M)$-cis-2, further photoisomerization in the same direction as the initial trans $\rightarrow$ cis photoisomerization is precluded by the steric strain associated with the equatorial orientation of the methyl substituents. However, the strain can be released in an energetically downhill thermal isomerization step that inverts the helicities of the two motor halves from $M$ to $P$ and restores the preferred axial orientations of the methyl groups to produce the $(P, P)$-cis-2 isomer. This step effectively blocks photoinduced reverse rotations of the $(M, M)$-cis-2 isomer and completes the first $180^{\circ}$ of the rotation. 
In the third step, irradiation of the $(P, P)$-cis-2 isomer with UV light induces a cis $\rightarrow$ trans photoisomerization to produce the $(M, M)$-trans-1 isomer, in which the methyl groups are once again trapped in a strained equatorial orientation. Analogous to the first step, this reaction also changes the helicities of the motor halves from $P$ to $M$. The preceding spontaneous thermal step ensures that this photoisomerization occurs in the same direction as the initial trans $\rightarrow$ cis photoisomerization. Thus, the two photoisomerizations occur in a unidirectional way and produce rotary motion.

Finally, the fourth step in analogous to the second one involves an energetically downhill thermal isomerization that restores the $P$ helicities of the motor halves and the preferred axial orientations of the methyl groups. This step completes the full $360^{\circ}$ rotation and returns the system to the initial $(P, P)$-trans- 1 state.

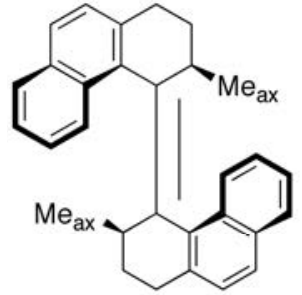

$(P, P)$-trans-1

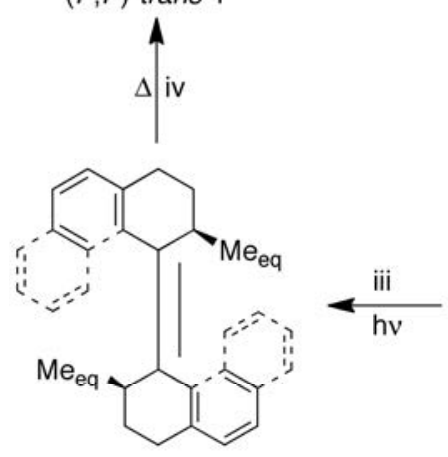

$(M, M)$-trans-1

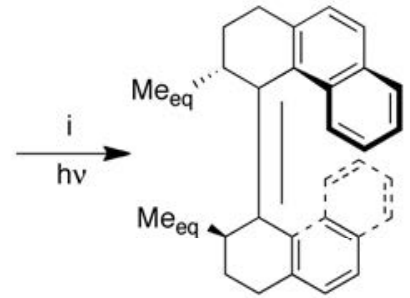

$(M, M)$ - cis-2

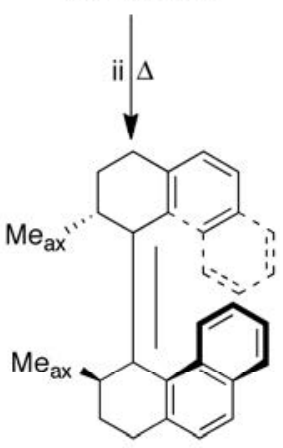

$(P, P)$-cis-2

Figure 3.1. Overall rotary cycle of molecular motor 1.

Overall, the rotary motion produced by overcrowded alkene-based molecular motors is governed by the steric interactions between the motor halves in the fjord regions. These interactions ensure that the photoisomerizations are unidirectional and that the thermal isomerizations are exergonic. ${ }^{6,22}$ As the photoisomerizations are 
known to proceed extremely fast (in less than $10 \mathrm{ps),}{ }^{26,30}$ it is the thermal isomerizations that limit the overall rotational frequencies that can be attained by the motors. ${ }^{6,22}$ Specifically, for motor $\mathbf{1}$, it was experimentally observed that the fourth step is the rate-determining one of the rotary cycle with a free-energy barrier of $\sim 105$ $\mathrm{kJ} \mathrm{mol}^{-1}$ and a half-life of $\sim 440 \mathrm{~h}$ under ambient conditions. ${ }^{10}$

In order to harness the potential of molecular motors in nanotechnology, ${ }^{11-16} \mathrm{a}$ key requirement is that the motors are able to achieve high rotational frequencies under ambient conditions. To this end, major experimental efforts have been invested in accelerating the rate-limiting thermal isomerizations of overcrowded alkene-based molecular motors. ${ }^{9,10,17,18,20,22-25,27,28,31}$ These efforts have resulted in the development of motors with very small thermal free-energy barriers $\left(\sim 35 \mathrm{~kJ} \mathrm{~mol}^{-1}\right),{ }^{23}$ so that they are able to reach MHz-rotational frequencies under suitable irradiation conditions. ${ }^{9,23}$ One example of such a motor, ${ }^{23}$ hereafter referred to as motor 2 , is shown in Figure 3.2. However, the mechanisms of the thermal isomerizations of this motor are largely unknown, which makes it difficult to further improve its performance. In this regard, following a comprehensive investigation of the rotary cycle of motor $\mathbf{1}$ in Paper I of the thesis for benchmarking the accuracy of various density functionals, the mechanisms of the thermal isomerizations of motor 2 were explored in full detail in Paper II using DFT methods. Moreover, through computational design of several new molecular motors of this type, Paper II proposes a steric approach to further accelerate the thermal isomerizations of motor 2. In a similar vein, Papers III and IV, in turn, propose electronic (Paper III) and conformational (Paper IV) approaches to substantially improve the performance of the thermal isomerizations of motors of this type.

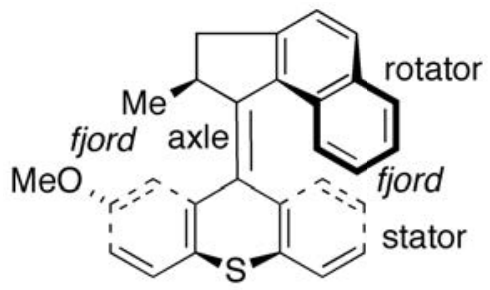

2

Figure 3.2. Chemical structure of the MHz-capable rotary molecular motor 2. 


\subsubsection{Paper I}

In Paper I, the full rotary cycle of motor $\mathbf{1}$ was modeled using DFT and TD-DFT methods for ground and excited states, respectively. Particularly, using the $\omega$ B97X-D and B3LYP functionals (and also several other functionals for benchmark purposes), it was found that these functionals are able to reproduce crystallographic and absorption data for the $(P, P)$-trans-1 and $(P, P)$-cis-2 isomers of this motor with reasonable accuracy. Furthermore, the geometric relaxation of the $(P, P)$-trans-1 and $(P, P)$-cis-2 isomers from the vertically excited Franck-Condon (FC) points in the lowest excited singlet state $\left(\mathrm{S}_{1}\right)$ predicted by TD-DFT involves considerable torsional motion around the central carbon-carbon double bond (see Figure 3.3). Pleasingly, this photoinduced torsional motion was found to proceed in the same direction $\mathrm{CCW}$ - for both the isomers, which is in consistent with the fact that the photoisomerizations of these isomers afford unidirectional rotary motion. The photoisomerizations of the $(P, P)$-trans-1 and $(P, P)$-cis-2 isomers were found to produce the $(M, M)$-cis-2 and $(M, M)$-trans-1 isomers, respectively.
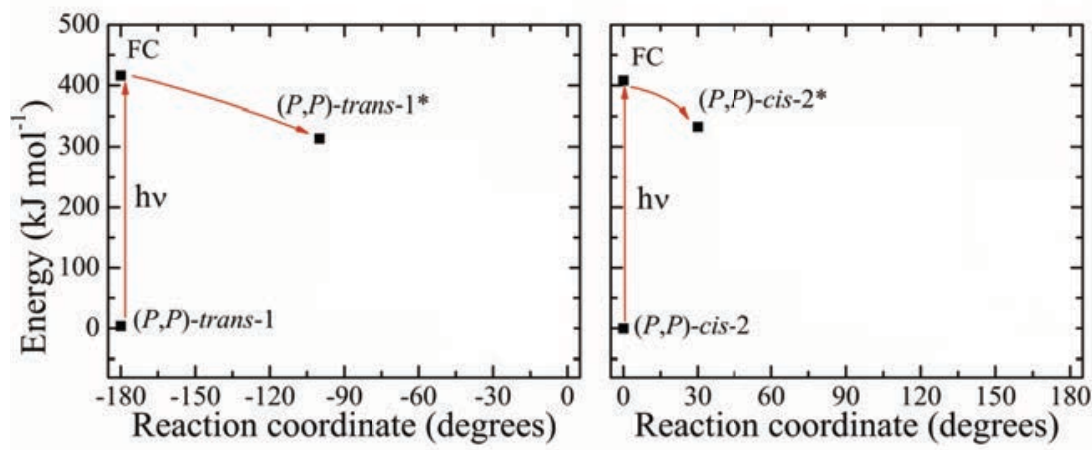

Figure 3.3. FC relaxation processes of the $(P, P)$-trans-1 and $(P, P)$-cis-2 isomers of motor 1 predicted by TD-DFT.

Turning to the thermal isomerizations of the $(M, M)$-cis-2 and $(M, M)$-trans-1 photoproduct isomers, the calculations summarized in Figure 3.4 predict that these processes proceed in two steps with the formation of an intermediate in which the helicity of one of the motor halves is inverted from $M$ to $P$. This supports the available experimental data on motors of this type. ${ }^{10}$ Furthermore, the calculations 
predict that the thermal isomerization of the $(M, M)$-trans-1 isomer is the overall ratelimiting process of the rotary cycle with a free-energy barrier of $\sim 136 \mathrm{~kJ} \mathrm{~mol}^{-1}$, which is in reasonable accordance with the corresponding experimental estimate of $\sim 107 \mathrm{~kJ}$ $\mathrm{mol}^{-1}$. $^{10}$

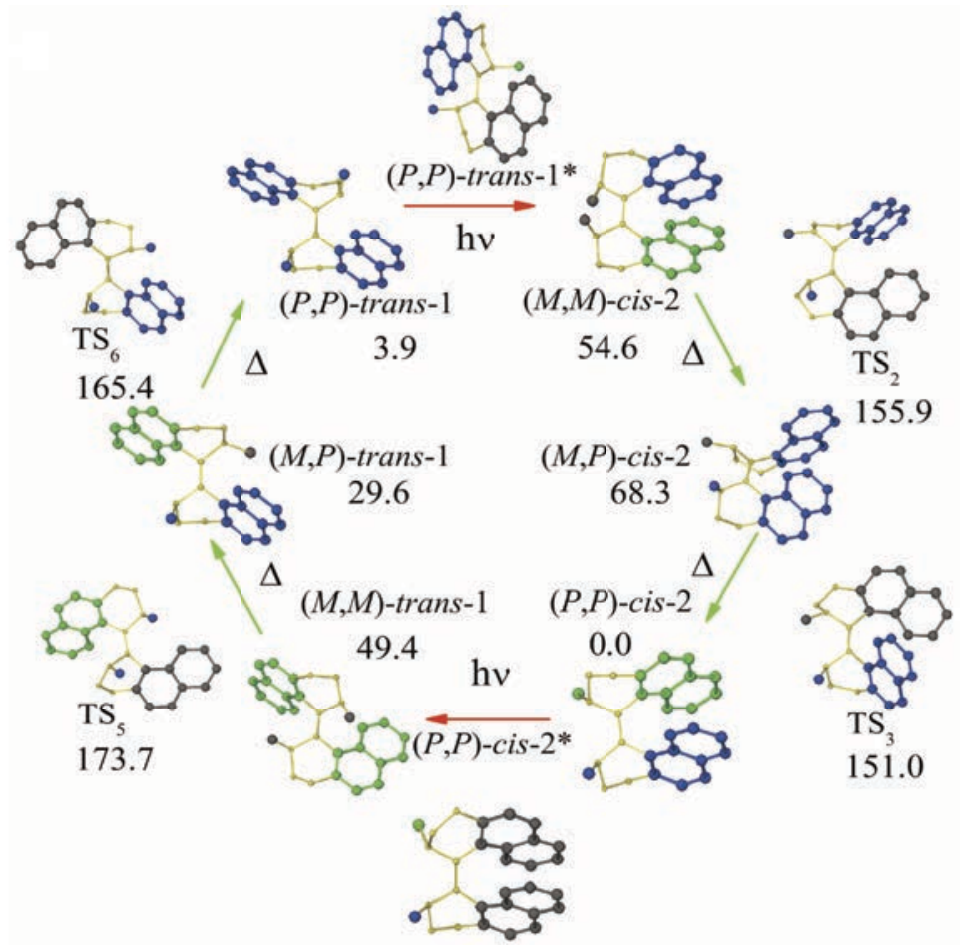

Figure 3.4. $\omega \mathrm{B} 97 \mathrm{X}-\mathrm{D}$ relative free energies of different stationary points along the rotary cycle of motor 1 with pointing-out, pointing-in, and planar orientations of the naphthyl rings indicated by blue, green, and black colors, respectively. Reproduced from Paper I with permission from The Royal Society of Chemistry.

Investigating also the thermal isomerizations of the MHz-capable molecular motor 2 to further assess the performance of $\omega \mathrm{B} 97 \mathrm{X}-\mathrm{D}$ and $\mathrm{B} 3 \mathrm{LYP}$, it was found that these methods can accurately reproduce the experimental $\sim 35 \mathrm{~kJ} \mathrm{~mol}^{-1}$ thermal barrier for this motor. ${ }^{23}$ Finally, using these methods, it was suggested that replacing the methyl group of motor $\mathbf{2}$ with a methoxy group is a possible strategy to accelerate the thermal isomerizations of motor 2 by $15-20 \mathrm{~kJ} \mathrm{~mol}^{-1}$. 


\subsubsection{Paper II}

In Paper II, the thermal isomerizations of motor $\mathbf{2}$ were explored comprehensively using DFT methods. Specifically, in addition to the $\omega$ B97X-D and B3LYP functionals used extensively in Paper I, three other functionals (PBE0, ${ }^{117}$ M06-2X ${ }^{118}$ and CAM-B3LYP) were also employed for this purpose. Through these calculations, a three-step mechanism was proposed for the thermal isomerizations. The calculated free-energy profiles (with $\omega \mathrm{B} 97 \mathrm{X}-\mathrm{D}$ ) for this mechanism are shown in Figure 3.5. Moreover, a possible side reaction that can impede the rotary rate was predicted.

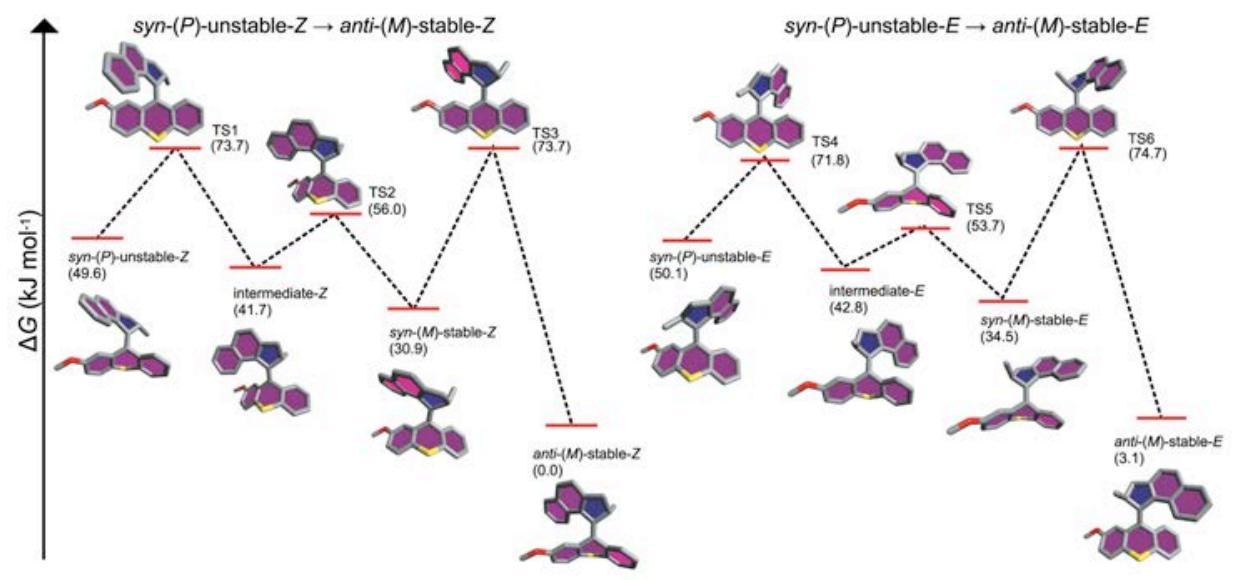

Figure 3.5. Free-energy profiles for the thermal isomerizations of motor 2 . Reproduced from Paper III with permission from Springer.

From Figure 3.5, it should be noted that the $Z$ and $E$ isomers exist in several possible conformations that differ in following aspects. First, the orientation of the rotator methyl substituent can either be equatorial ("unstable") or axial ("stable"). Second, the relative folding of the stator and rotator with respect to the plane containing the central double bond and the stereocenter (hereafter referred to as olefinic plane) can either be syn or anti. Finally, the helicity of the rotator can either be $P$ or $M$ (or absent). Overall, the two most stable species are the anti-(M)-stable- $Z$ and $\operatorname{anti-}(M)$-stable- $E$ isomers and two least stable ones are the syn- $(P)$-unstable- $Z$ and syn-(P)-unstable- $E$ isomers. While the former are the light-absorbing isomers, ${ }^{23}$ the latter were found to be the photoisomerization products. 
Focusing on mechanistic aspects, the first two steps of the thermal isomerizations of both the syn- $(P)$-unstable- $Z$ and $s y n-(P)$-unstable- $E$ photoproduct isomers involve an inversion of the rotator helicity from $P$ to $M$ and a change in orientation of the methyl substituent from equatorial to axial. In the third and final step, the stator undergoes a ring flip relative to the olefinic plane that changes the relative stator-rotator folding from syn to anti. This step is the rate-determining step for the thermal isomerizations of both the isomers. Furthermore, it was found that the calculated thermal free-energy barriers of this step given by $\omega$ B97X-D agree to within 6-8 $\mathrm{kJ} \mathrm{mol}^{-1}$ of available kinetic data for these processes. ${ }^{23}$

Having accurately reproduced available kinetic data for the thermal barriers of motor 2, eight new potential rotary molecular motors (3-10) were then designed by replacing the stator methoxy and rotator methyl substituents of motor $\mathbf{2}$ with groups of varying steric bulkiness, as shown in Figure 3.6. The thermal isomerizations of these motors were explored exclusively with $\omega \mathrm{B} 97 \mathrm{X}-\mathrm{D}$. Besides its accurate predictions for motor 2, another reason for employing $\omega$ B97X-D is that it includes empirical atomatom dispersion corrections ${ }^{119,120}$ and is therefore well suited for the description of intramolecular interactions between the stator and rotator.

Stator-substituted

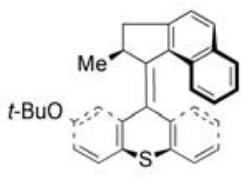

3

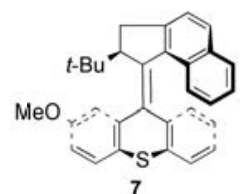

7

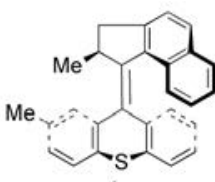

4

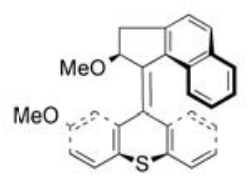

8

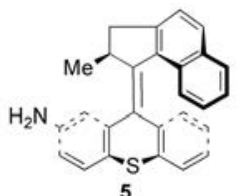

5

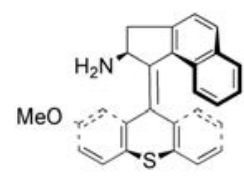

9

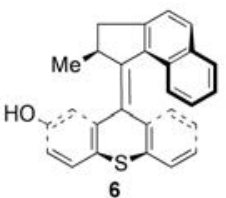

6

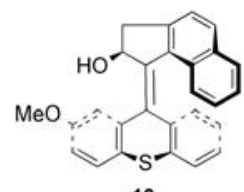

10

Figure 3.6. Chemical structures of potential rotary molecular motors considered in Paper II.

From the calculations, it was predicted that the thermal isomerizations of motors 3-10 follow the same exact three-step mechanism as motor 2. Overall, it was found that the thermal isomerizations of motor $\mathbf{2}$ can be accelerated by modulating the 
steric bulkiness of the rotator substituent. On the other hand, the stator substituent was found to have almost no influence on the thermal isomerization rates.

Specifically, the free-energy barrier of the third step of motor $\mathbf{2}$ is lowered by up to $13-19 \mathrm{~kJ} \mathrm{~mol}^{-1}$ with a smaller $\left(\mathrm{OMe}, \mathrm{NH}_{2}\right.$ and $\mathrm{OH}$ rotator groups of motors 8-10) and raised by up to $29-35 \mathrm{~kJ} \mathrm{~mol}^{-1}$ with a bulkier ( $t$-Bu rotator group of motor 7) substituent than methyl group. On the other hand, the second step shows contrasting behavior to the third step (see Figure 3.7). This can be understood in terms of how the amount of steric repulsion in the TSs of the two steps compares with that of in the associated reactant species. Overall, it was found that the rate-determining barriers of motors 8-10 are up to $17 \mathrm{~kJ} \mathrm{~mol}^{-1}$ smaller than those of the MHz-capable motor $2 .^{23}$ Therefore, these motors hold promise to reach beyond-MHz rotational frequencies, provided the irradiation step is not rate limiting. Altogether, Paper II reports a steric approach to accelerate the thermal isomerizations of the overcrowded alkene-based molecular motors.

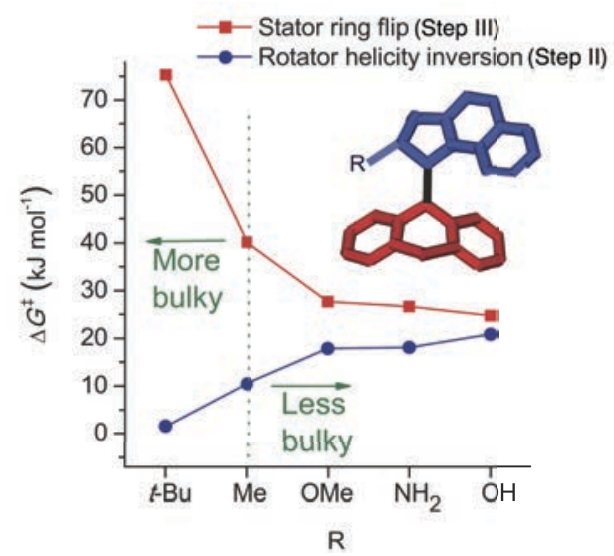

Figure 3.7. Free-energy barriers for the second and third steps in the thermal isomerization of the syn- $(P)$-unstable- $Z$ isomer of different rotator-substituted motors. Reproduced from Paper II with permission from the PCCP Owner Societies.

\subsubsection{Paper III}

As it was found in Paper II that the steric bulkiness of the stator substituent exerts virtually no influence on the rates of the thermal isomerizations, in Paper III the focus was instead put on the electronic character of the stator substituent. Specifically, 
DFT methods was used to investigate whether the thermal isomerizations of motors 2 and $\mathbf{8}$ (studied in Paper II), and a variant of motor $\mathbf{2}$ obtained by replacing the rotator methyl group with a nitro group (referred to as motor 11), can be accelerated by introducing either electron-donating or electron-withdrawing stator substituents in conjugation with the central olefinic bond. Considering these motors as the reference motors (see Figure 3.8), electron-donating methoxy and dimethylamino groups were introduced at the C3 and C6 positions of the stator to obtain motors 12-14 and electron-withdrawing nitro and cyano groups were introduced at the same positions to obtain motors $\mathbf{1 5}-\mathbf{1 7}$.

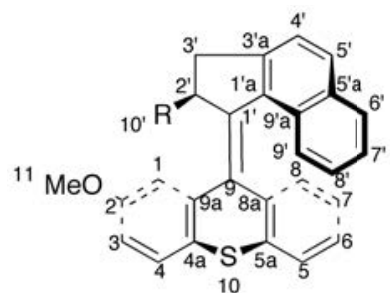

$$
\begin{aligned}
& \text { 2: } R=\mathrm{Me} \\
& \text { 11: } R=\mathrm{NO}_{2} \\
& \text { 8: } R=O M e
\end{aligned}
$$

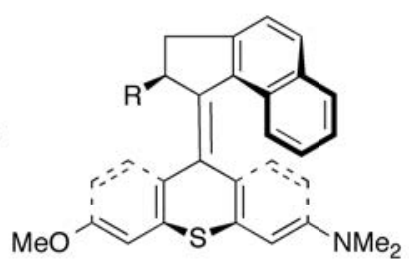

12: $\mathrm{R}=\mathrm{Me}$

13: $\mathrm{R}=\mathrm{NO}_{2}$

14: $\mathrm{R}=\mathrm{OM}$<smiles>[R7]C1Cc2ccc3ccccc3c2/C1=C1\c2ccc(C#N)cc2Sc2cc([N+](=O)[O-])ccc21</smiles>

15: $\mathrm{R}=\mathrm{Me}$ 16: $\mathrm{R}=\mathrm{NO}_{2}$ 17: $\mathrm{R}=\mathrm{OM}$

2: reference motor for 12 and 15

11: reference motor for 13 and 16

8: reference motor for 14 and 17

Figure 3.8. Chemical structures of potential rotary molecular motors considered in Paper III and the details of the reference motors.

Continuing to use $\omega \mathrm{B} 97 \mathrm{X}-\mathrm{D}$ to explore the thermal isomerizations, the same three-step mechanism documented for motor $\mathbf{2}$ in Paper II was implicated also for all the other motors studied in Paper III. Furthermore, while it was found that the electron-withdrawing stator substituents of motors 15-17 has no influence on the rates of the thermal isomerizations, it was demonstrated that the electron-donating stator substituents of motors 12-14 are able to reduce the free-energy barriers of the rate-determining third step for the reference motors by up to $18 \mathrm{~kJ} \mathrm{~mol}^{-1}$ without substantially altering the barriers of the other two steps (see Figure 3.9). Accordingly, motors 12-14 appear capable of achieving post-MHz rotational frequencies under suitable irradiation conditions. 

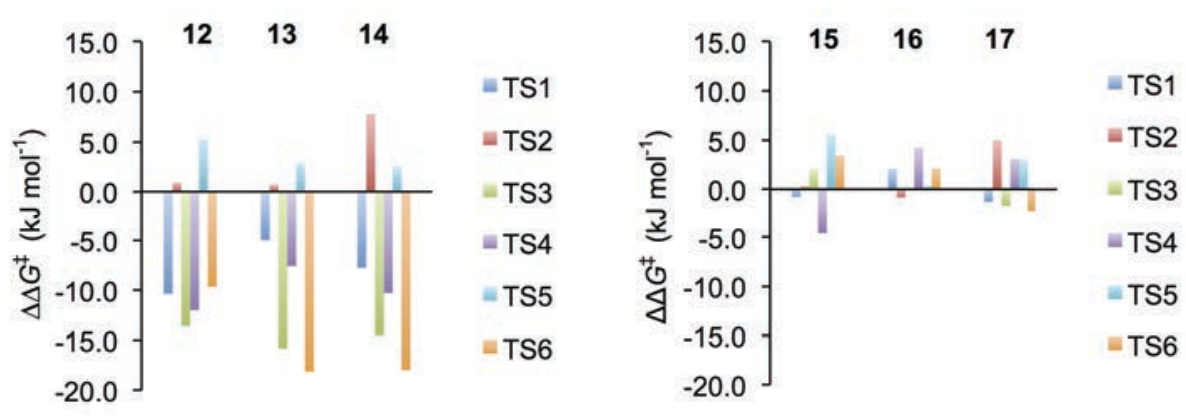

Figure 3.9. Thermal free-energy barriers for motors $\mathbf{1 2}-\mathbf{1 4}$ and $\mathbf{1 5}-\mathbf{1 7}$ relative to those for the reference motors. Positive (negative) values indicate increase (decrease) in the barriers for these motors relative to those for the reference motors.

Finally, exploring the origin of the catalytic effect by the electron-donating stator substituents, it was found that these groups (unlike the electron-withdrawing ones) exert a favorable steric influence on the critical third step of the isomerizations. Interestingly, such influence is not based on resonance-induced elongation of the central carbon-carbon double bond in the TSs for the third step (that would have stabilized the TSs sterically), but is a consequence of a destabilizing steric effect on the associated reactants of motors 12-14. This effect arises from a flatter (more conjugated) stator in the reactants of these motors relative to that in the reactants of the reference motors. Overall, Paper III proposes an electronic approach to control the critical steric interactions of the overcrowded alkene-based molecular motors.

\subsubsection{Paper IV}

In Paper IV, the aim was to improve the thermal isomerization performance of the MHz-capable motor 2 and two of its structural analogues ${ }^{9}$ through a redesign of these motors wherein the sizes of the stator and rotator halves are substantially reduced. To this end, three new motors $\mathbf{1 8 - 2 0}$ featuring a synthetically-viable indanylidene rotator and a six-membered diene ring stator, but differing in the stator bridging atom $(\mathrm{X}=$ $\mathrm{CH}_{2}$ or $\mathrm{O}$ or $\mathrm{S}$ ) were designed (see Figure 3.10). 


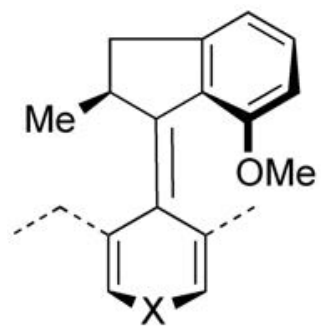

18: $\mathrm{X}=\mathrm{CH}_{2}$

19: $X=O$

20: $X=S$

Figure 3.10. Chemical structures of potential rotary molecular motors considered in Paper IV.

First, by performing multi-configurational quantum chemical calculations using the CASSCF method, it was demonstrated that the photoisomerizations of the light-absorbing anti- $(M)$-stable- $E$ and anti- $(M)$-stable- $Z$ isomers of motors 18-20 occur in a unidirectional fashion and produce rotary motion. Furthermore, it was found that these isomerizations produce, respectively, the $(P)$-unstable- $Z$ and $(P)$ unstable- $E$ isomers as photoproducts.

Next, by using DFT methods (mainly $\omega$ B97X-D) to explore the thermal isomerizations of the $(P)$-unstable- $Z$ and $(P)$-unstable- $E$ isomers, one stepwise and one concerted mechanism were identified for these processes. The calculated freeenergy profiles for these mechanisms are shown in Figure 3.11. As can be seen from these profiles, the stepwise mechanism proceeds in two steps via a syn-folded intermediate, where the first step involves an inversion of the rotator helicity from $P$ to $M$ and the second step a change in relative folding of the stator and rotator from syn to anti. The concerted mechanism, in turn, proceeds without any intermediate(s) but via a highly asynchronous TS, in which a change in the helicity of the rotator is not manifested.

From the calculations, it was observed that the free-energy barriers of both the mechanisms vary considerably between motors 18-20. This variation can be explained in terms of how a change in the size of the stator bridging atom affects the steric repulsion in reactants and TSs. For motors $\mathbf{1 8}$ and 19, the stepwise mechanism was found to be the kinetically preferred one with a rate-determining barrier of 18 and 
$41 \mathrm{~kJ} \mathrm{~mol}^{-1}$, respectively. For motor 20, the two mechanisms have similar barriers of $23-24 \mathrm{~kJ} \mathrm{~mol}^{-1}$.

$(P)$-unstable- $Z \rightarrow$ anti-(M)-stable-Z

$(P)$-unstable- $E \rightarrow$ anti-(M)-stable- $E$
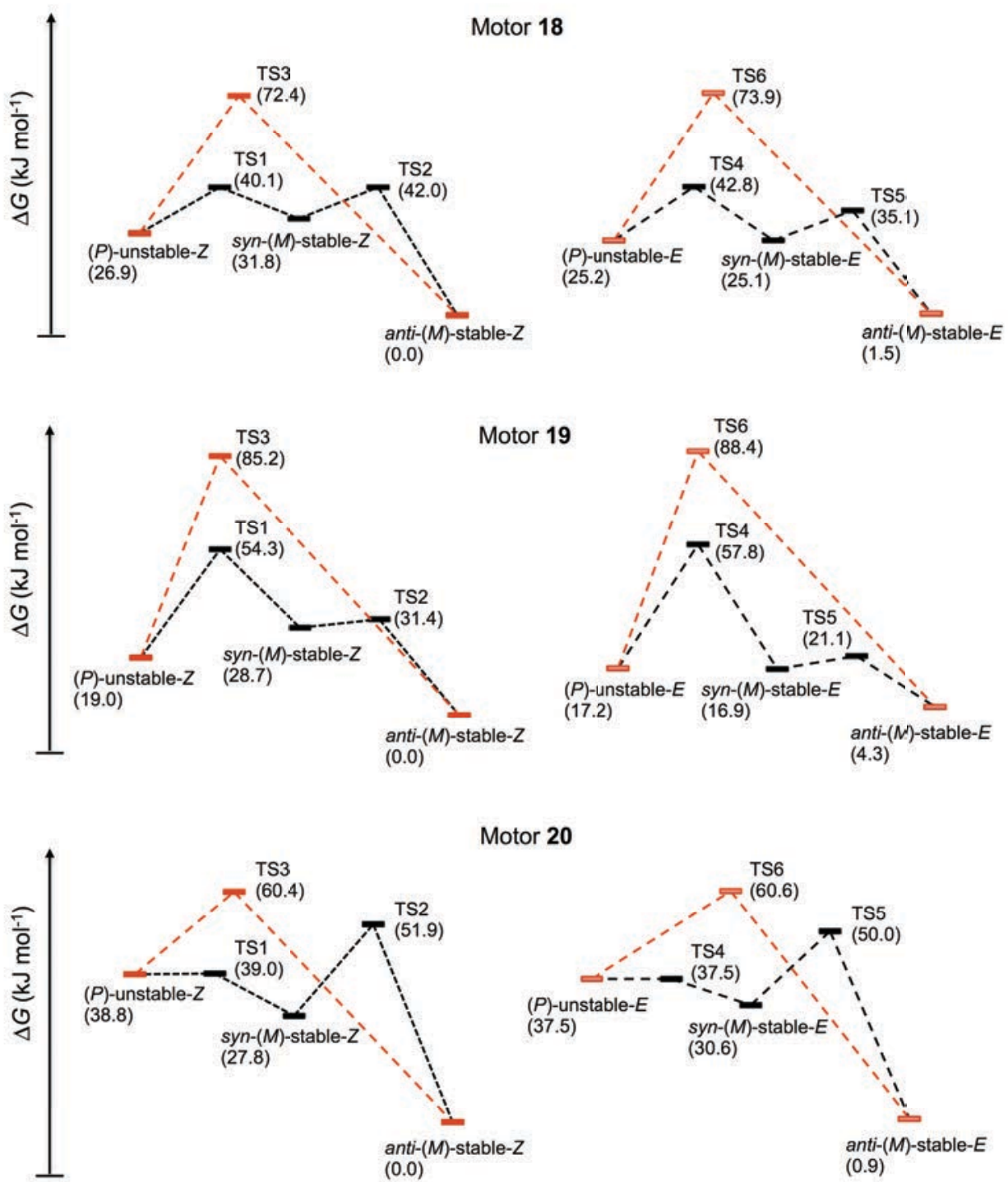

Figure 3.11. Free-energy profiles for stepwise and concerted mechanisms for the thermal isomerizations of motors 18-20. 
The rate-determining barriers of motors 18 and 20 are $19-25 \mathrm{~kJ} \mathrm{~mol}^{-1}$ smaller than the corresponding $43 \mathrm{~kJ} \mathrm{~mol}^{-1}$ barrier computed for the MHz-capable motor 2 at the same level of theory. Additionally, the thermal isomerizations of all the three motors involve fewer steps than those of motor $\mathbf{2}$ and occur without the negative impact of the competing side reaction predicted for motor $\mathbf{2}$ in Paper II. The reason for such mechanistic differences was found to be that the $Z$ and $E$ isomers of motors 18-20 exist in fewer conformations than those of motor 2. Overall, Paper IV suggests a conformational approach to improve the performance of the thermal isomerizations of the overcrowded alkene-based molecular motors.

\subsection{Protonated Schiff Bases}

A cis $\rightarrow$ trans photoisomerization around a carbon-carbon double bond in the retinal protonated Schiff-base (RPSB) chromophore (see Figure 3.12) of the visual pigment rhodopsin initiates the process of vision. ${ }^{1,2}$ This reaction has several interesting features. First, the photoinduced rotation has a preferred direction, owing to the chirality of the rhodopsin environment, and occurs with a high quantum yield of $\sim 0.67 .^{1,121}$ Second, the photoisomerization is completed within $200 \mathrm{fs}^{122-124} \mathrm{In}$ contrast, the photoisomerization steps of overcrowded alkene-based motors typically occurs in 1-10 $\mathrm{ps}^{26,30}$ and with quantum yields of $0.1-0.3{ }^{30,125}$ Therefore, systems capable of mimicking the photoisomerization features of the RPSB chromophore can potentially yield a new class of rotary molecular motors capable of surpassing the performance of the overcrowded alkene-based motors both in terms of rate and efficiency.

Indanylidene-pyrrolinium (IP) molecular switches, shown in Figure 3.12, were originally synthesized ${ }^{126}$ to mimic the photoisomerization features of the RPSB chromophore. Recently, Olivucci, Léonard and Gindensperger (hereafter OLG) have demonstrated that the IP framework must be made chiral to exert control over the direction of rotation. ${ }^{127}$ Specifically, by incorporating a chiral cyclopentenylidene moiety into a protonated Schiff-base framework, they showed that unidirectional rotary motion can be achieved in this system from two photoisomerization steps alone, without the need of the thermal isomerization steps. Moreover, they found that point chirality is essential for this capability. In Paper $\mathbf{V}$, the protonated Schiff-base 
design is further generalized by demonstrating that it can also produce unidirectional rotary motion in a purely photochemical fashion in the absence of point chirality.

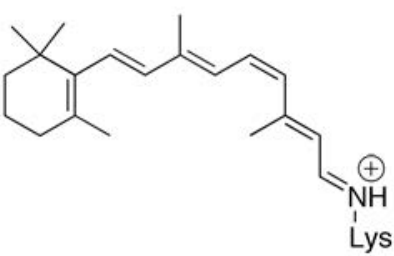

RPSB

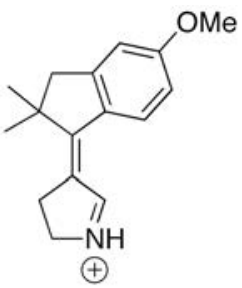

IP switch

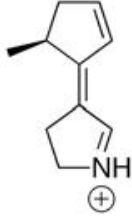

OLG motor

Figure 3.12. Chemical structures of the RPSB chromophore, IP switch and OLG motor.

\subsubsection{Paper V}

In Paper $\mathbf{V}$, the nearly flat cyclopentenylidene moiety of the OLG motor was replaced with a strained cyclohexenylidene moiety. Furthermore, the single stereocenter of the OLG motor, which was deemed essential for unidirectional rotary motion, ${ }^{127}$ was removed by replacing the methyl substituent of cyclopentenylidene with a hydrogen atom in the new motor, hereafter denoted motor $\mathbf{I}$ and shown in Figure 3.13. As a result of the ring strain in cyclohexenylidene, both the $E$ and $Z$ isomers (about the central carbon-carbon double bond) of I have two possible orientations for the $5^{\prime}$ carbon relative to the approximate plane of other atoms of the ring - outward (out) and inward (in). Overall, there are four possible isomers of $\mathbf{I}$, labeled $E_{\text {out }}, E_{\text {in }}, Z_{\text {out }}$ and $Z_{\text {in }}$, and constituting two pairs of axially chiral enantiomers: $E_{\text {out }} / E_{\text {in }}$ and $Z_{\text {out }} / Z_{\text {in }}$.

First, the equilibrium ground-state $\left(\mathrm{S}_{0}\right)$ geometries of all isomers of $\mathbf{I}$ were obtained with the multi-configurational CASSCF method. The helical pretwist of the central carbon-carbon double bond in the resulting geometries was characterized by the $\Delta \omega$ dihedral angle, where $\omega$ is the mean of the $\theta$ and $\theta^{\prime}$ dihedral angles (see Figure $3.13)$ and $\Delta \omega$ is the signed deviation of $\omega$ from the untwisted $\omega$ values of 0 or $\pm 180^{\circ}$. It was found that the $E_{\text {out }}$ and $Z_{\text {in }}$ isomers show a positive helical pretwist of $\sim 3^{\circ}$ and the $E_{\text {in }}$ and $Z_{\text {out }}$ isomers a negative helical pretwist of similar magnitude. Therefore, it 
is of interest to assess if the positive or negative sign of helical pretwist influences the direction $(\mathrm{CW}$ or $\mathrm{CCW})$ of the photoinduced rotation.

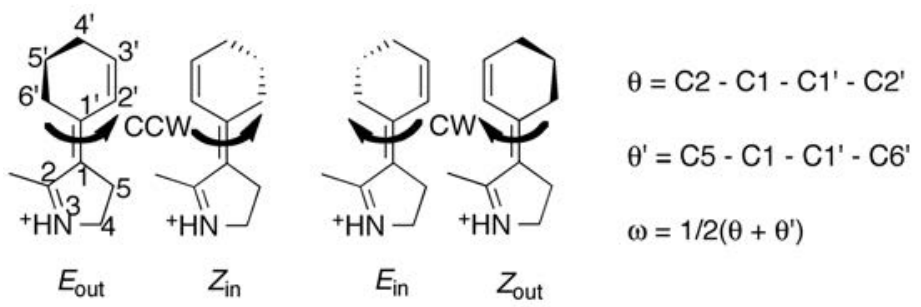

Figure 3.13. Chemical structures of the $E_{\text {out }}, Z_{\text {in }}, E_{\text {in }}$ and $Z_{\text {out }}$ isomers of motor $\mathbf{I}$ and definition of relevant dihedral angles.

The photoisomerizations of the isomers of I were first modeled by carrying out minimum energy path calculations using SA-CASSCF with equal (0.5) weights for $S_{0}$ and $S_{1}$. For each geometry along the MEP, singlepoint calculations were performed with CASPT2 to account for dynamic correlation effects. These calculations showed that the photoinduced torsional motion is CCW (toward increasing values of $\omega$ ) for the $E_{\text {out }}$ and $Z_{\text {in }}$ isomers, and $\mathrm{CW}$ (toward decreasing values of $\omega$ ) for the $E_{\text {in }}$ and $Z_{\text {out }}$ isomers.

Next, the photoisomerizations were then modeled by performing NAMD simulations using SA-CASSCF with identical weights for $S_{0}$ and $S_{1}$. The simulations were run maximally for 700 fs with 200 different initial nuclear configurations and velocities. A successful trajectory of the simulations was defined as one that changes $\omega$ by $180^{\circ}$ with respect to the starting configuration within 700 fs. The percentage of successful trajectories, distributions of associated photoisomerization times (PITs), and the corresponding changes in $\omega$ values are shown in Figure 3.14.

Figure 3.14 shows that the percentage of successful trajectories is very high ( $\geq 88 \%$ ) for each photoisomerization. Furthermore, all successful trajectories of each isomer were found to evolve in one direction only, $\mathrm{CCW}$ for the $E_{\text {out }}$ and $Z_{\text {in }}$ isomers and $\mathrm{CW}$ for the $E_{\text {in }}$ and $Z_{\text {out }}$ isomers. As the $E_{\text {out }}$ isomerizes into $Z_{\text {in }}$ (and vice versa) and $E_{\text {in }}$ isomerizes into $Z_{\text {out }}$ (and vice versa), the $\left[E_{\text {out }}, Z_{\text {in }}\right]$ and $\left[E_{\text {in }}, Z_{\text {out }}\right]$ pairs of isomers of I produce $360^{\circ}$ unidirectional rotary motion. Notably, the <PIT> values were found to be consistently small, only 260-270 fs. 
Finally, it was demonstrated that the unidirectional rotary motion produced by the $\left[E_{\text {out }}, Z_{\text {in }}\right]$ and $\left[E_{\text {in }}, Z_{\text {out }}\right]$ pairs of isomers of $\mathbf{I}$ is not a consequence of the small positive or negative helical pretwists in these isomers, but rather a manifestation of the inherent asymmetry of the strained cyclohexenylidene. Overall, Paper $\mathbf{V}$ proposes a novel way to achieve photoinduced unidirectional rotary motion in Schiff-base motors without the need of point chirality and helical motifs.

(a)

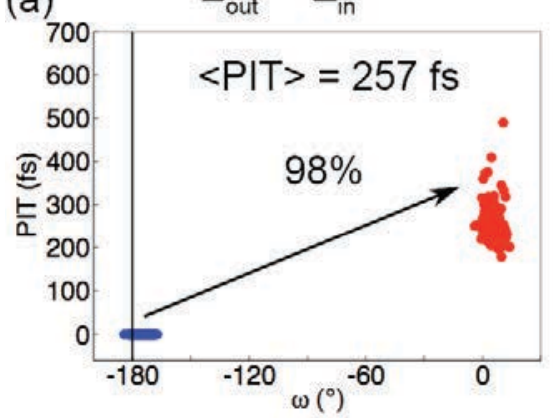

(d)

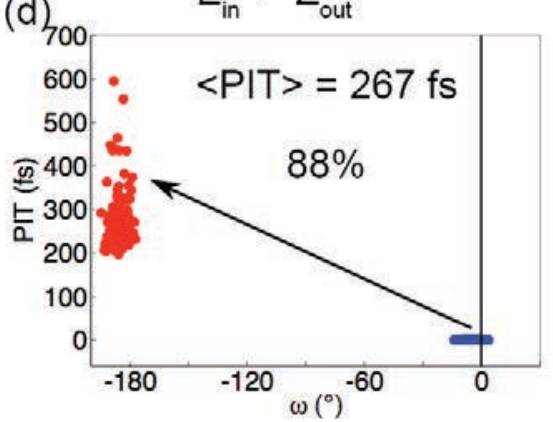

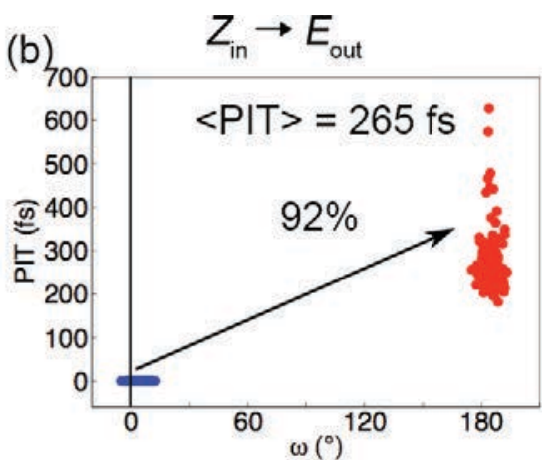

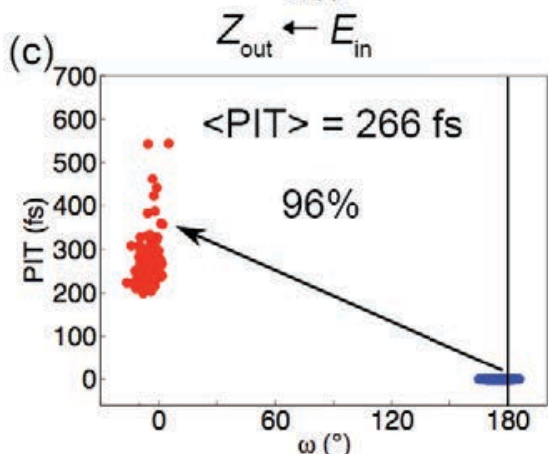

Figure 3.14. Distributions of PITs for successful (red circles) $1-E_{\text {out }} \rightarrow \mathbf{1}-Z_{\text {in }}\left(\right.$ a), $1-Z_{\text {in }}$ $\rightarrow 1-E_{\text {out }}(\mathrm{b}), \mathbf{1}-E_{\text {in }} \rightarrow 1-Z_{\text {out }}$ (c) and $1-Z_{\text {out }} \rightarrow 1-E_{\text {in }}$ (d) trajectories and the corresponding changes in $\omega$ relative to the starting configurations (blue circles). 


\section{Excited-State Studies of Organic Chromophores}

\subsection{Composite Procedures}

Benchmark studies are essential in quantum chemistry for assessing the performance of different available methods in modeling different types of chemical systems and problems. Such studies are particularly important for studying excited electronic states due to the challenges associated with describing these states by quantum chemical calculations. A common approach in benchmarks of excited-state methods is to compare the computed $\Delta E_{\mathrm{ve}}$ energies with either experimental absorption maxima or $\Delta E_{\mathrm{ve}}$ energies obtained from higher-level correlation treatments. Such comparisons rely essentially on two key assumptions. First, it is assumed that molecular geometry is unchanged during the electronic transition (the Franck-Condon principle). Second, it is assumed that the absorption maximum is not influenced by vibrational and rotational effects.

A comprehensive approach for comparing computational excited-state data with spectroscopic data is to simulate vibrationally resolved electronic absorption spectra. Although such simulations have been reported by a few research groups, ${ }^{128-}$ 133 they are not easily affordable in broad benchmark studies that consider a large number of chemical systems and a variety of excited-state methods. Instead, some excited-state benchmarks have rather focused on $\Delta E_{\text {ad }}$ or $\Delta E_{00}$ energies, ${ }^{88-92,134-137}$ which represent a compromise between $\Delta E_{\mathrm{ve}}$-based benchmarks and vibronic simulations. Although such studies increase the computational cost by requiring excited-state geometry optimizations and frequency calculations, the required calculations are nowadays feasible due to the efficient implementations of analytic gradients for many excited-state methods. ${ }^{60,75-79,93,138-141}$

Papers VI and VII contribute to the benchmarking of excited-state methods from a different perspective. Specifically, instead of focusing on how well different excited-state methods reproduce $\Delta E_{\mathrm{ad}}$ or $\Delta E_{00}$ energies, these papers focus on the 
design and assessment of a composite procedure that combines TD-DFT and CC2 calculations to obtain $\Delta E_{00}$ energies of $\mathrm{CC} 2$ quality in a cost-effective way. Specifically, in this procedure, TD-DFT was used instead of CC2 to estimate the $\Delta \Delta E_{\text {ad }}$ (excited-state relaxation energy) and $\Delta \Delta E_{00}$ (difference in ZPVE corrections of the excited state and the ground state) terms of $\Delta E_{00}=\Delta E_{\mathrm{ve}}+\Delta \Delta E_{\mathrm{ad}}+\Delta \Delta E_{00}$. A prerequisite for the success of such a procedure is that the $\Delta \Delta E_{\text {ad }}$ and $\Delta \Delta E_{00}$ terms must be relatively insensitive to the choice of quantum chemical method. Therefore, Paper VI investigates the extent of method sensitivity of these two terms by considering a benchmark set comprising both organic and inorganic systems. Having thereby found in Paper VI that the method insensitivity of the $\Delta \Delta E_{\text {ad }}$ and $\Delta \Delta E_{00}$ terms is more pronounced for organic systems than for inorganic systems, Paper VII considers only organic systems to assess the performance of a composite procedure that combines TD-DFT and CC2 calculations.

\subsubsection{Paper VI}

In Paper VI, a benchmark set of 79 different molecules covering organic chromophores such as carbonyl compounds, aromatic hydrocarbons and aromatic heterocyclic compounds, as well as several inorganic compounds, was considered as detailed in Table 4.1. Employing seven different density functionals (BP86, B3LYP, PBE0, M06-2X, M06-HF, ${ }^{142}$ CAM-B3LYP and $\omega$ B97X-D) and two ab initio methods (CIS and CC2), the $\Delta E_{\mathrm{ve}}, \Delta E_{\mathrm{ad}}$ and $\Delta E_{00}$ energies for a total of 96 different excited states, classified into nine different groups, were computed. Most of these states are single-reference valence excited states, which are often well described by TD-DFT and CC2 methods. As stated above, the main objective of Paper VI was to investigate to what extent the $\Delta \Delta E_{\mathrm{ad}}$ and $\Delta \Delta E_{00}$ energy differences are sensitive to the choice of quantum chemical method, rather than to assess the accuracy with which the methods reproduce the experimental $\Delta E_{00}$ energies. In this regard, it is interesting to note that these terms were found to vary by as little as $\sim 0.1 \mathrm{eV}$ between different quantum chemical methods for chromophores of the photoactive yellow protein and the green fluorescent protein. ${ }^{134}$ 


\section{Excited-State Studies of Organic Chromophores}

\begin{tabular}{|c|c|c|c|c|c|c|c|c|}
\hline group $\mathrm{I}^{g}$ & $\mathrm{id}^{b}$ & states & group II ${ }^{h}$ & $\mathrm{id}^{b}$ & states & 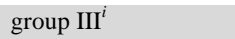 & $\mathrm{id}^{b}$ & states \\
\hline $\mathrm{Li}_{2}$ & 1 & $\mathrm{~S}_{1} 1^{1} \Sigma^{+}{ }_{\mathrm{u}}$ & $\mathrm{BeO}$ & 1 & $S_{1} 1^{1} \Pi$ & $\mathrm{HCN}$ & 1 & $S_{1} 1^{1} A^{\prime \prime}$ \\
\hline \multirow[t]{3}{*}{$\mathrm{N}_{2}$} & 2 & $\mathrm{~S}_{1} 1^{1} \Delta_{\mathrm{u}}$ & $\mathrm{BH}$ & 2 & $\mathrm{~S}_{1} 1^{\mathrm{l} \Pi}$ & $\mathrm{HCP}$ & 2 & $S_{1} 2^{1} A^{\prime}$ \\
\hline & 3 & $\mathrm{~S}_{2} 1^{1} \Pi_{\mathrm{g}}$ & $\mathrm{BF}$ & 3 & $\mathrm{~S}_{1} 1^{\mathrm{l} \Pi}$ & $\mathrm{CS}_{2}$ & 3 & $\mathrm{~T}_{2} 1^{3} \mathrm{~A}_{2}$ \\
\hline & 4 & $\mathrm{~T}_{1} 1^{3} \Pi_{\mathrm{g}}$ & $\mathrm{CO}$ & 4 & $S_{1} 1^{1} \Pi$ & $\mathrm{NH}_{3}$ & 4 & $S_{1} 1^{1} A^{\prime \prime}{ }_{2}$ \\
\hline $\mathrm{Mg}_{2}$ & 5 & $\mathrm{~S}_{1} 1^{1} \Sigma^{+} \mathrm{u}$ & & 5 & $\mathrm{~T}_{1} 1^{3} \Pi$ & & 5 & $\mathrm{~S}_{2} 3^{1} \mathrm{~A}$ \\
\hline $\mathrm{P}_{2}$ & 6 & $S_{1} 1^{1} \Pi_{g}$ & $\mathrm{NH}$ & 6 & $\mathrm{~T}_{1} 1^{3} \Pi$ & $\mathrm{SiF}_{2}$ & 6 & $\mathrm{~S}_{1} 1^{1} \mathrm{~B}_{1}$ \\
\hline \multirow[t]{3}{*}{$\mathrm{Cu}_{2}$} & 7 & $\mathrm{~S}_{1} 1^{1} \Sigma^{+}{ }_{\mathrm{u}}^{+}$ & $\mathrm{SiO}$ & 7 & $\mathrm{~S}_{1} 1^{1} \Pi$ & & & \\
\hline & 8 & $\mathrm{~S}_{2} 1^{1} \Pi_{\mathrm{u}}$ & $\mathrm{CuH}$ & 8 & $S_{1} 2^{1} \Sigma^{+}$ & & & \\
\hline & & & AsF & 9 & $\mathrm{~T}_{1} 1^{3} \Pi$ & & & \\
\hline group $\mathrm{IV}^{j}$ & $\mathrm{id}^{b}$ & states & group $\mathrm{V}^{k}$ & $\mathrm{id}^{b}$ & states & group VI ${ }^{l}$ & $\mathrm{id}^{b}$ & states \\
\hline \multirow[t]{2}{*}{ formaldehyde } & 1 & $\mathrm{~S}_{1} 1^{1} \mathrm{~A}_{2}$ & dichlorocarbene & 1 & $\mathrm{~S}_{1} 1^{1} \mathrm{~B}_{1}$ & benzene & 1 & $\mathrm{~S}_{1} 1^{1} \mathrm{~B}_{2 \mathrm{u}}$ \\
\hline & 2 & $\mathrm{~T}_{1} 1^{3} \mathrm{~A}_{2}$ & aminomethane & 2 & $S_{1} 2^{1} A^{\prime}$ & toluene & 2 & $S_{1} 1^{1} A^{\prime \prime}$ \\
\hline \multirow[t]{2}{*}{ methanethial } & 3 & $\mathrm{~S}_{1} 1^{1} \mathrm{~A}_{2}$ & & 3 & $\mathrm{~S}_{2} 1^{1} \mathrm{~A}^{\prime \prime}$ & styrene & 3 & $\mathrm{~S}_{1} 2^{1} \mathrm{~A}$ \\
\hline & 4 & $\mathrm{~T}_{1} 1^{3} \mathrm{~A}_{2}$ & aminoethane & 4 & $S_{1} 2^{1} A^{\prime}$ & 1,4-diethynylbenzene & 4 & $\mathrm{~S}_{1} 1^{1} \mathrm{~B}_{1 \mathrm{u}}$ \\
\hline formic acid & 5 & $\mathrm{~S}_{1} 2^{1} \mathrm{~A}$ & acetylene & 5 & $\mathrm{~S}_{1} 2^{1} \mathrm{~A}$ & biphenyl & 5 & $\mathrm{~S}_{1} 1^{1} \mathrm{~B}_{1}$ \\
\hline acetaldehyde & 6 & $\mathrm{~S}_{1} 2^{1} \mathrm{~A}$ & cyanoacetylene & 6 & $S_{1} 1^{1} A^{\prime \prime}$ & biphenylene & 6 & $\mathrm{~S}_{1} 1^{1} \mathrm{~B}_{1 \mathrm{u}}$ \\
\hline \multirow[t]{2}{*}{ acrolein } & 7 & $S_{1} 1^{1} A^{\prime \prime}$ & hexatriene & 7 & $\mathrm{~S}_{1} 1^{1} \mathrm{~B}_{\mathrm{u}}$ & trans-stilbene & 7 & $\mathrm{~S}_{1} 2^{1} \mathrm{~A}$ \\
\hline & 8 & $\mathrm{~T}_{1} 1^{3} \mathrm{~A}^{\prime \prime}$ & octatetraene & 8 & $\mathrm{~S}_{1} 1^{1} \mathrm{~B}_{\mathrm{u}}$ & naphthalene & 8 & $\mathrm{~S}_{1} 1^{1} \mathrm{~B}_{1 \mathrm{u}}$ \\
\hline propynal & 9 & $\mathrm{~S}_{1} 2^{1} \mathrm{~A}$ & & & & anthracene & 9 & $\mathrm{~S}_{1} 1^{\mathrm{l}} \mathrm{B}_{1 \mathrm{u}}$ \\
\hline acetone & 10 & $\mathrm{~S}_{1} 2^{1} \mathrm{~A}$ & & & & azulene & 10 & $\mathrm{~S}_{1} 1^{1} \mathrm{~B}_{2}$ \\
\hline \multirow[t]{2}{*}{ thioacetone } & 11 & $\mathrm{~S}_{1} 2^{1} \mathrm{~A}$ & & & & fluorene & 11 & $\mathrm{~S}_{1} 2^{1} \mathrm{~A}_{1}$ \\
\hline & 12 & $\mathrm{~T}_{1} 1^{3} \mathrm{~A}$ & & & & pyrene & 12 & $\mathrm{~S}_{1} 2^{1} \mathrm{~A}$ \\
\hline glyoxal & 13 & $\mathrm{~S}_{1} 1^{1} \mathrm{~A}_{\mathrm{u}}$ & & & & terylene & 13 & $\mathrm{~S}_{1} 2^{1} \mathrm{~A}^{\prime}$ \\
\hline oxalyfluoride & 14 & $\mathrm{~S}_{1} 1^{1} \mathrm{~A}_{\mathrm{u}}$ & & & & & & \\
\hline group VII $^{m}$ & $\operatorname{id}^{b}$ & states & group VIII $^{n}$ & $\mathrm{id}^{b}$ & states & group IX ${ }^{o}$ & $\mathrm{id}^{b}$ & states \\
\hline phenol & 1 & $\mathrm{~S}_{1} 2^{1} \mathrm{~A}$ & pyridine & 1 & $\mathrm{~S}_{1} 2^{1} \mathrm{~A}$ & 2-cyclopenten-1-one & 1 & $\mathrm{~S}_{1} 2^{1} \mathrm{~A}$ \\
\hline anisol & 2 & $\mathrm{~S}_{1} 2^{1} \mathrm{~A}$ & pyrimidine & 2 & $\mathrm{~S}_{1} 2^{1} \mathrm{~A}$ & 1,4-benzoquinone & 2 & $S_{1} 1^{1} B_{1 g}$ \\
\hline aniline & 3 & $\mathrm{~S}_{1} 2^{1} \mathrm{~A}$ & & 3 & $\mathrm{~S}_{3} 2^{1} \mathrm{~A}_{2}$ & & 3 & $\mathrm{~S}_{3} 1^{1} \mathrm{~B}_{3 \mathrm{~g}}$ \\
\hline \multirow[t]{3}{*}{ benzaldehyde } & 4 & $S_{1} 1^{1} A^{\prime \prime}$ & 2-chloropyrimidine & 4 & $S_{1} 2^{1} A^{\prime}$ & $\mathrm{DBH}^{f}$ & 4 & $\mathrm{~S}_{1} 2^{1} \mathrm{~A}$ \\
\hline & 5 & $\mathrm{~S}_{2} 2^{1} \mathrm{~A}^{\prime}$ & pyridone lactim & 5 & $\mathrm{~S}_{1} 2^{1} \mathrm{~A}$ & $\begin{array}{l}\text { 1,6-epoxy-10- } \\
\text { annulene }\end{array}$ & 5 & $\mathrm{~S}_{1} \quad 1^{1} \mathrm{~B}_{1}$ \\
\hline & 6 & $\mathrm{~T}_{1} 1^{3} \mathrm{~A}^{\prime \prime}$ & pyridone lactam & 6 & $\mathrm{~S}_{1} 2^{1} \mathrm{~A}$ & & & \\
\hline benzonitrile & 7 & $\mathrm{~S}_{1} 1^{\mathrm{I}} \mathrm{B}_{2}$ & tetrazine & 7 & $\mathrm{~S}_{1} 2^{1} \mathrm{~A}$ & & & \\
\hline 4-aminobenzonitrile & 8 & $\mathrm{~S}_{1} 1^{1} \mathrm{~B}_{1}$ & indole & 8 & $S_{1} 2^{1} A^{\prime}$ & & & \\
\hline DMA-benzonitrile $^{c}$ & 9 & $\mathrm{~S}_{1} 1^{1} \mathrm{~B}$ & 7-azaindole & 9 & $\mathrm{~S}_{1} 2^{1} \mathrm{~A}$ & & & \\
\hline hydroquinone & 10 & $\mathrm{~S}_{1} 1^{1} \mathrm{~B}_{\mathrm{u}}$ & cinnoline & 10 & $S_{1} 1^{1} A^{\prime \prime}$ & & & \\
\hline $\begin{array}{l}1,4- \\
\text { phenylenediamine }\end{array}$ & 11 & $\mathrm{~S}_{1} 2^{1} \mathrm{~A}$ & quinoline & 11 & $\mathrm{~S}_{1} 2^{1} \mathrm{~A}$ & & & \\
\hline DMA-aniline $^{d}$ & 12 & $\mathrm{~S}_{1} 2^{1} \mathrm{~A}$ & & 12 & $\mathrm{~T}_{1} 1^{3} \mathrm{~A}^{\prime}$ & & & \\
\hline methyl 4-coumarate & 13 & $\mathrm{~S}_{1} 2^{1} \mathrm{~A}$ & quinoxaline & 13 & $\mathrm{~S}_{1} 2^{1} \mathrm{~A}$ & & & \\
\hline \multirow[t]{5}{*}{ DMA-cyanostilbene ${ }^{e}$} & 14 & $\mathrm{~S}_{1} 2^{1} \mathrm{~A}$ & & $\begin{array}{l}14 \\
15\end{array}$ & $\begin{array}{l}\mathrm{S}_{3} 2^{1} \mathrm{~A}_{1} \\
\mathrm{~T}_{1} 1^{3} \mathrm{~A}\end{array}$ & & & \\
\hline & & & $\beta$-dinaphthyleneoxide & 16 & $\mathrm{~S}_{1} 2^{1} \mathrm{~A}$ & & & \\
\hline & & & syn-coumarin 153 & 17 & $\mathrm{~S}_{1} 2^{1} \mathrm{~A}$ & & & \\
\hline & & & porphyrin & 18 & $\mathrm{~S}_{1} 1^{1} \mathrm{~B}_{1 \mathrm{u}}$ & & & \\
\hline & & & merocyanine dye & 19 & $\mathrm{~S}_{1} 2^{1} \mathrm{~A}$ & & & \\
\hline
\end{tabular}

${ }^{a}$ The molecules in groups IV-IX are depicted in Figure S1 of the Supporting Information. Symmetry labels reflect molecular geometries after excited-state relaxation. ${ }^{b}$ Label used in figures. ${ }^{c} 4$-(dimethylamino)benzonitrile. ${ }^{d} 4$-(dimethylamino)aniline. ${ }^{e} 4-$ (dimethylamino)-4'-cyanostilbene. ${ }^{f} 2,3$-diazabicyclo[2.2.1]-hept-2-ene. ${ }^{g}$ Group I: inorganic molecules - homodiatomics. ${ }^{h}$ Group II: inorganic molecules - heterodiatomics. ${ }^{i}$ Group III: inorganic molecules - polyatomics. ${ }^{j}$ Group IV: carbonyl and thiocarbonyl compounds. ${ }^{k}$ Group V: hydrocarbons. ${ }^{l}$ Group VI: aromatic hydrocarbons. ${ }^{m}$ Group VII: substituted aromatic hydrocarbons. ${ }^{n}$ Group VIII: heterocyclic aromatic compounds. ${ }^{o}$ Group IX: cyclic non-aromatic compounds. 


\section{Excited-State Studies of Organic Chromophores}

Figure 4.1 compares the standard deviations (SDs) for the calculated $\Delta E_{\mathrm{ve}}$ energies and corresponding data for the calculated $\Delta \Delta E_{\mathrm{ad}}$ and $\Delta \Delta E_{00}$ energy differences for all the nine groups of the benchmark set. Overall, it was found that while the average standard deviation (ASD) for $\Delta E_{\mathrm{ve}}$ energies (over all the nine groups) between different methods is $0.39 \mathrm{eV}$, the corresponding standard deviations for $\Delta \Delta E_{\mathrm{ad}}$ and $\Delta \Delta E_{00}$ energy differences are merely 0.10 and $0.02 \mathrm{eV}$, respectively. Notably, from Table 4.2, the ASDs for calculating $\Delta \Delta E_{\mathrm{ad}}$ for organic systems (0.08 $\mathrm{eV}$ for Groups IV-IX) were found to be smaller than those for inorganic systems ( $0.15 \mathrm{eV}$ for Groups I-III). These results indicate that accurate estimates of the $\Delta \Delta E_{\mathrm{ad}}$ and $\Delta \Delta E_{00}$ energy differences, particularly for organic systems, are well-amenable to cheap methods.
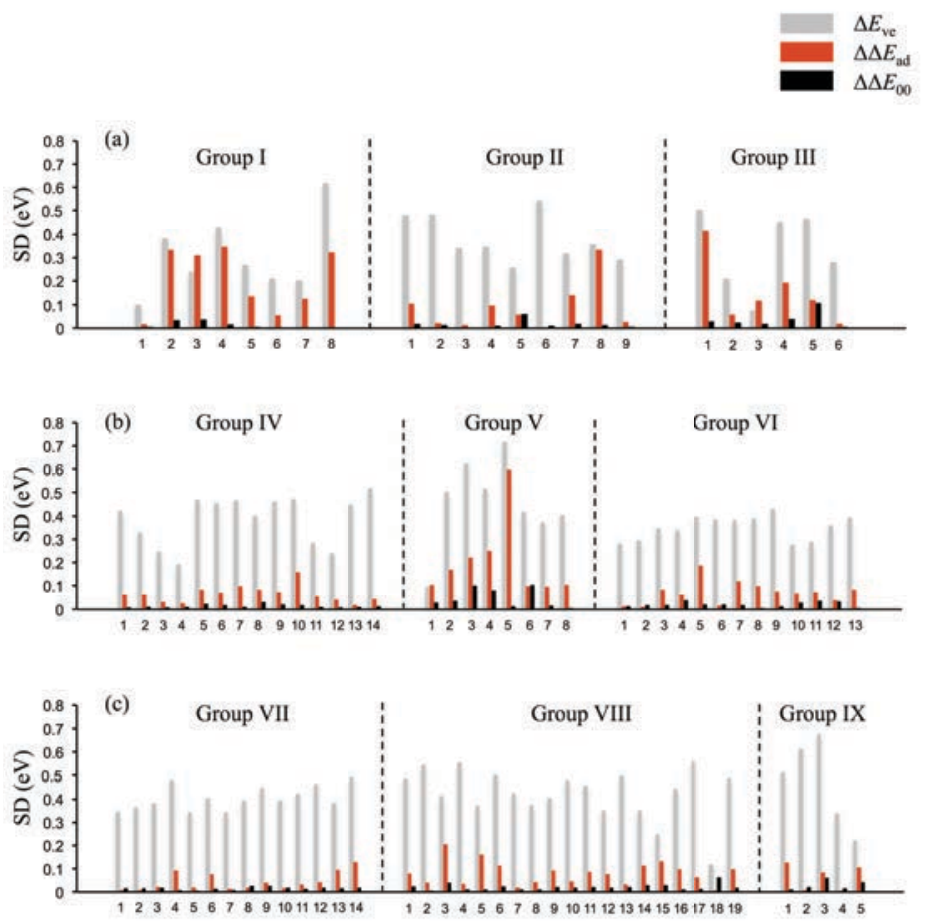

Figure 4.1. SDs for calculated $\Delta E_{\mathrm{ve}}$ energies and $\Delta \Delta E_{\mathrm{ad}}$ and $\Delta \Delta E_{00}$ energy differences of individual states in groups I-III (a), IV-VI (b), and VII-IX (c) of the benchmark set using the BP86, B3LYP, PBE0, M06-2X, M06-HF, CAM-B3LYP, WB97X-D, CIS and CC2 methods. Reprinted from Paper VI with permission from The Journal of Physical Chemistry A. Copyright 2014 American Chemical Society. 


\begin{tabular}{|c|c|c|c|c|c|c|}
\hline \multirow[b]{2}{*}{ group $(s)$} & \multicolumn{2}{|c|}{$\Delta E_{\mathrm{ve}}$} & \multicolumn{2}{|c|}{$\Delta \Delta E_{\mathrm{ad}}$} & \multicolumn{2}{|c|}{$\Delta \Delta E_{00}$} \\
\hline & $\mathrm{ASD}^{a}$ & $\operatorname{GMaxAD}^{a}$ & $\mathrm{ASD}^{a}$ & $\operatorname{GMaxAD}^{a}$ & $\mathrm{ASD}^{a}$ & $\operatorname{GMaxAD}^{a}$ \\
\hline I & 0.31 & 1.62 & 0.21 & 1.20 & 0.01 & 0.13 \\
\hline II & 0.38 & 1.93 & 0.09 & 1.11 & 0.02 & 0.20 \\
\hline III & 0.33 & 1.84 & 0.15 & 1.68 & 0.04 & 0.36 \\
\hline IV & 0.39 & 1.94 & 0.06 & 0.56 & 0.02 & 0.12 \\
\hline V & 0.46 & 2.65 & 0.20 & 1.97 & 0.05 & 0.34 \\
\hline VI & 0.35 & 1.50 & 0.07 & 0.64 & 0.02 & 0.13 \\
\hline VII & 0.40 & 1.87 & 0.04 & 0.44 & 0.02 & 0.09 \\
\hline VIII & 0.42 & 2.20 & 0.08 & 0.74 & 0.02 & 0.23 \\
\hline IX & 0.47 & 2.29 & 0.07 & 0.40 & 0.03 & 0.17 \\
\hline I-III & 0.34 & 1.93 & 0.15 & 1.68 & 0.02 & 0.36 \\
\hline IV-IX & 0.41 & 2.65 & 0.08 & 1.97 & 0.02 & 0.34 \\
\hline I-IX & 0.39 & 2.65 & 0.10 & 1.97 & 0.02 & 0.36 \\
\hline
\end{tabular}

As for the performance of the different methods in calculating $\Delta E_{00}$ energies, the smallest errors relative to experimental data were obtained with $\mathrm{CC} 2$ with a mean absolute error (MAE) of $0.19 \mathrm{eV}$ for the full benchmark set. Particularly, CC2 showed better performance for the organic systems (MAE of $0.15 \mathrm{eV}$ ) than the inorganic ones (MAE of $0.31 \mathrm{eV}$ ). Considering this and the finding that the method insensitivity of the $\Delta \Delta E_{\text {ad }}$ and $\Delta \Delta E_{00}$ energy differences is more prominent for the organic systems, Paper VII considers a benchmark set of only organic systems.

\subsubsection{Paper VII}

In Paper VII, a benchmark set of 66 single-reference excited states of medium-sized and large organic chromophores (shown in Table 4.3), comprising mainly aromatic hydrocarbons and aromatic heterocyclic compounds, was considered. The $\Delta E_{\mathrm{ve}}, \Delta E_{\mathrm{ad}}$ and $\Delta E_{00}$ energies for all the excited states were computed using seven different density functionals (BP86, B3LYP, PBE0, M06-2X, M06-HF, CAM-B3LYP and $\omega \mathrm{B} 97 \mathrm{X}-\mathrm{D})$ and two ab initio methods (CIS and CC2) in a method-consistent way, wherein the geometries, electronic energies and ZPVE corrections needed to calculate the $\Delta E_{\mathrm{ve}}, \Delta E_{\mathrm{ad}}$ and $\Delta E_{00}$ energies at a particular level of theory were obtained at that very level. 
Table 4.3. Groups of molecules and excited states in the benchmark set. ${ }^{a}$ Reprinted from Paper VII with permission from Molecular Physics. Copyright 2016 Taylor \& Francis Group Ltd.

\begin{tabular}{|c|c|c|c|c|c|}
\hline Group I ${ }^{b}$ & $\mathrm{Label}^{c}$ & State & Group II ${ }^{d}$ & Label $^{c}$ & State \\
\hline pyrazine & 1 & $\mathrm{~S}_{1} 1^{1} \mathrm{~A}_{\mathrm{u}}$ & tetrafluorobenzene & 1 & $\mathrm{~S}_{1} 1^{1} \mathrm{~B}_{1}$ \\
\hline 2,6-difluoropyridine & 2 & $\mathrm{~S}_{1} 1^{1} \mathrm{~B}_{2}$ & benzonitrile & 2 & $\mathrm{~S}_{1} 1^{1} \mathrm{~B}_{2}$ \\
\hline 2-fluoropyridine & 3 & $\mathrm{~S}_{1} 2^{1} \mathrm{~A}^{\prime}$ & $o$-fluorophenol, cis & 3 & $\mathrm{~S}_{1} 2^{1} \mathrm{~A}$ \\
\hline 2-hydroxypyridine & 4 & $\mathrm{~S}_{1} 2^{1} \mathrm{~A}^{\prime}$ & $o$-fluorophenol, trans & 4 & $\mathrm{~S}_{1} 2^{1} \mathrm{~A}$ \\
\hline 2-pyridone & 5 & $\mathrm{~S}_{1} 2^{1} \mathrm{~A}^{\prime}$ & $m$-fluorophenol, cis & 5 & $\mathrm{~S}_{1} 2^{1} \mathrm{~A}$ \\
\hline 2-methylpyrimidine & 6 & $\mathrm{~S}_{1} 1^{1} \mathrm{~A}^{\prime \prime}$ & $m$-fluorophenol, trans & 6 & $\mathrm{~S}_{1} 2^{1} \mathrm{~A}^{\prime}$ \\
\hline 5-methylpyrimidine & 7 & $\mathrm{~S}_{1} 1^{1} \mathrm{~A}^{\prime \prime}$ & $p$-fluorophenol & 7 & $\mathrm{~S}_{1} 2^{1} \mathrm{~A}^{\prime}$ \\
\hline 7-azaindole & 8 & $\mathrm{~S}_{1} 2^{1} \mathrm{~A}^{\prime}$ & phenylacetylene & 8 & $\mathrm{~S}_{1} \quad 1^{1} \mathrm{~B}_{2}$ \\
\hline 7-hydroxyquinoline, cis & 9 & $\mathrm{~S}_{1} 2^{1} \mathrm{~A}^{\prime}$ & aniline & 9 & $\mathrm{~S}_{1} 1^{1} \mathrm{~A}^{\prime \prime}$ \\
\hline 7-hydroxyquinoline, trans & 10 & $\mathrm{~S}_{1} 2^{1} \mathrm{~A}$ & catechol & 10 & $\mathrm{~S}_{1} 2^{1} \mathrm{~A}$ \\
\hline 2-hydroxyquinoline, enol & 11 & $\mathrm{~S}_{1} 2^{1} \mathrm{~A}^{\prime}$ & resorcinol, isomer 1 & 11 & $\mathrm{~S}_{1} 2^{1} \mathrm{~A}$ \\
\hline 2-hydroxyquinoline, keto & 12 & $\mathrm{~S}_{1} 2^{1} \mathrm{~A}^{\prime}$ & resorcinol, isomer 2 & 12 & $\mathrm{~S}_{1} 1^{1} \mathrm{~B}_{2}$ \\
\hline dibenzofuran & 13 & $\mathrm{~S}_{1} 2^{1} \mathrm{~A}_{1}$ & resorcinol, isomer 3 & 13 & $\mathrm{~S}_{1} 1^{1} \mathrm{~A}^{\prime \prime}$ \\
\hline pyrrolo[3,2- $h]$ quinoline & 14 & $\mathrm{~S}_{1} 2^{1} \mathrm{~A}^{\prime}$ & hydroquinone, cis & 14 & $\mathrm{~S}_{1} 2^{1} \mathrm{~A}_{1}$ \\
\hline carbazole & 15 & $\mathrm{~S}_{1} 2^{1} \mathrm{~A}_{1}$ & hydroquinone, trans & 15 & $\mathrm{~S}_{1} 1^{1} \mathrm{~B}_{\mathrm{u}}$ \\
\hline tryptamine, A-ph ${ }^{e}$ & 16 & $\mathrm{~S}_{1} 2^{1} \mathrm{~A}$ & salicylic acid & 16 & $\mathrm{~S}_{1} 2^{1} \mathrm{~A}^{\prime}$ \\
\hline tryptamine, A-py ${ }^{f}$ & 17 & $\mathrm{~S}_{1} 2^{1} \mathrm{~A}$ & $o$-cresol, cis & 17 & $\mathrm{~S}_{1} 2^{1} \mathrm{~A}$ \\
\hline tryptamine, A-up ${ }^{g}$ & 18 & $\mathrm{~S}_{1} 2^{1} \mathrm{~A}$ & $o$-cresol, trans & 18 & $\mathrm{~S}_{1} 2^{1} \mathrm{~A}$ \\
\hline tryptamine, $\mathrm{Ph}$-ou $t^{h}$ & 19 & $\mathrm{~S}_{1} 2^{1} \mathrm{~A}$ & $m$-cresol, cis & 19 & $\mathrm{~S}_{1} 2^{1} \mathrm{~A}$ \\
\hline tryptamine, $\mathrm{Ph}-\mathrm{up}^{i}$ & 20 & $\mathrm{~S}_{1} 2^{1} \mathrm{~A}$ & $m$-cresol, trans & 20 & $\mathrm{~S}_{1} 2^{1} \mathrm{~A}^{\prime}$ \\
\hline tryptamine, Py-out ${ }^{j}$ & 21 & $\mathrm{~S}_{1} 2^{1} \mathrm{~A}$ & $p$-cresol & 21 & $\mathrm{~S}_{1} 2^{1} \mathrm{~A}$ \\
\hline tryptamine, Py-up ${ }^{k}$ & 22 & $\mathrm{~S}_{1} 2^{1} \mathrm{~A}$ & $o$-methoxyphenol & 22 & $\mathrm{~S}_{1} 2^{1} \mathrm{~A}$ \\
\hline porphycene & 23 & $\mathrm{~S}_{1} 2^{1} \mathrm{~A}^{\prime}$ & $m$-methoxyphenol, isomer 1 & 23 & $\mathrm{~S}_{1} 2^{1} \mathrm{~A}$ \\
\hline porphine & 24 & $\mathrm{~S}_{1} 1^{1} \mathrm{~B}_{1 \mathrm{u}}$ & $m$-methoxyphenol, isomer 2 & 24 & $\mathrm{~S}_{1} 2^{1} \mathrm{~A}$ \\
\hline chlorin & 25 & $\mathrm{~S}_{1} 1^{1} \mathrm{~B}_{2}$ & $p$-methoxyphenol, cis & 25 & $\mathrm{~S}_{1} 2^{1} \mathrm{~A}$ \\
\hline Zn-tetraphenylporphine & 26 & $\mathrm{~S}_{1} 1^{1} \mathrm{~B}_{2}$ & $p$-methoxyphenol, trans & 26 & $\mathrm{~S}_{1} 2^{1} \mathrm{~A}$ \\
\hline tetraphenylporphine & 27 & $\mathrm{~S}_{1} 1^{1} \mathrm{~B}$ & 1-naphthol, cis & 27 & $\mathrm{~S}_{1} 2^{1} \mathrm{~A}$ \\
\hline & & & 1-naphthol, trans & 28 & $\mathrm{~S}_{1} 2^{1} \mathrm{~A}^{\prime}$ \\
\hline & & & 2-naphthol, cis & 29 & $\mathrm{~S}_{1} 2^{1} \mathrm{~A}^{\prime}$ \\
\hline & & & 2-naphthol, trans & 30 & $\mathrm{~S}_{1} 2^{1} \mathrm{~A}^{\prime}$ \\
\hline & & & 5-methoxysalicylic acid & 31 & $\mathrm{~S}_{1} 2^{1} \mathrm{~A}^{\prime}$ \\
\hline & & & $m$-dimethoxybenzene, trans & 32 & $\mathrm{~S}_{1} 2^{1} \mathrm{~A}^{\prime}$ \\
\hline & & & 3P-propionic acid, ${ }^{1}$ gauche & 33 & $\mathrm{~S}_{1} 2^{1} \mathrm{~A}$ \\
\hline & & & 3P-propionic acid, ${ }^{1}$ anti & 34 & $\mathrm{~S}_{1} 1^{1} \mathrm{~A}^{\prime \prime}$ \\
\hline & & & fluorene & 35 & $\mathrm{~S}_{1} 1^{1} \mathrm{~B}_{2}$ \\
\hline & & & phenanthrene & 36 & $\mathrm{~S}_{1} 2^{1} \mathrm{~A}_{1}$ \\
\hline & & & 2,4,6,8-decatetraene & 37 & $\mathrm{~S}_{1} 1^{1} \mathrm{~B}_{\mathrm{u}}$ \\
\hline & & & tetracene & 38 & $\mathrm{~S}_{1} 1^{1} \mathrm{~B}_{1 \mathrm{u}}$ \\
\hline & & & perylene & 39 & $\mathrm{~S}_{1} 1^{1} \mathrm{~B}$ \\
\hline
\end{tabular}

${ }^{a}$ The molecules in two groups are depicted in Figures S1 and S3 of the Supplemental data. Symmetry labels reflect molecular geometries after excited-state relaxation. ${ }^{b}$ Group I: aromatic heterocyclic compounds. ${ }^{c}$ Label used in subsequent figures. ${ }^{d}$ Group II: aromatic (or aliphatic) hydrocarbons and substituted aromatic hydrocarbons. ${ }^{e}$ Anti-ph. ${ }^{f}$ Anti-py. ${ }^{g}$ Anti-up. ${ }^{h}$ gauche-phenyl-out. ${ }^{i}$ gauche-phenyl-up. ${ }^{j}$ gauche-pyrrole-out. ${ }^{k}$ gauche-pyrrole-up. ${ }^{\text {3-phenyl-1-propionic acid. }}$

From the calculations, it was found that the MAE over all the 66 states between the CC2 $\Delta E_{00}$ energies and experimentally available ones is only $0.11 \mathrm{eV}$, and corresponding maximum absolute error (MaxAE) is $0.22 \mathrm{eV}$. Such accuracy and robustness is not rivaled by any of the density functionals used, with the best- 
performing (in terms of MAE) functional B3LYP showing a MAE of $0.20 \mathrm{eV}$ and a MaxAE of $0.72 \mathrm{eV}$. It is therefore of interest to assess the performance of a composite procedure that combines CC2 and TD-DFT by exploiting the afore-mentioned method insensitivity of the $\Delta \Delta E_{\mathrm{ad}}$ and $\Delta \Delta E_{00}$ energy differences. To this end, it was investigated whether the 'true' $\mathrm{CC} 2 \Delta E_{00}$ energies, denoted $\Delta E_{00}(\mathrm{CC} 2)$ and obtained by explicitly using $\mathrm{CC} 2$ for all parts of the calculations as

$$
\Delta E_{00}(\mathrm{CC} 2)=\Delta E_{v e}(\mathrm{CC} 2)+\Delta \Delta E_{a d}(\mathrm{CC} 2)+\Delta \Delta E_{00}(\mathrm{CC} 2)
$$

can be approximated by calculations employing CC2 for the $\Delta E_{\mathrm{ve}}$ and $\Delta \Delta E_{\mathrm{ad}}$ terms or the $\Delta E_{\mathrm{ve}}$ term only, and a cheaper method ' $\mathrm{X}$ ' for the remaining term(s):

$$
\Delta E_{00}\left(\mathrm{CC} 2 / \mathrm{X}^{\prime}\right)=\Delta E_{v e}(\mathrm{CC} 2)+\Delta \Delta E_{a d}(\mathrm{CC} 2)+\Delta \Delta E_{00}(\mathrm{X})
$$

and

$$
\Delta E_{00}\left(\mathrm{CC} 2 / \mathrm{X}^{\prime \prime}\right)=\Delta E_{v e}(\mathrm{CC} 2)+\Delta \Delta E_{a d}(\mathrm{X})+\Delta \Delta E_{00}(\mathrm{X})
$$

respectively. The absolute errors (AEs) of $\Delta E_{00}\left(\mathrm{CC} 2 / \mathrm{X}^{\prime}\right)$ and $\Delta E_{00}\left(\mathrm{CC} 2 / \mathrm{X}^{\prime \prime}\right)$ relative to true $\Delta E_{00}(\mathrm{CC} 2)$ for each of the 66 states are shown in Figures 4.2 and 4.3, respectively.

Starting with the results obtained with the $\Delta E_{00}\left(\mathrm{CC} 2 / \mathrm{X}^{\prime}\right)$ scheme shown in Figure 4.2, in which TD-DFT or CIS was used instead of CC2 for calculating the $\Delta \Delta E_{00}$ term, the results showed that the MAEs introduced by this scheme are very small, a mere $0.03-0.04 \mathrm{eV}$. Thus, for almost all the states considered in this work it is definitely possible to employ TD-DFT or CIS for calculating the $\Delta \Delta E_{00}$ term with almost no affect on the accuracy of the final $\Delta E_{00}$ energies.

Turning to the results obtained with the $\Delta E_{00}\left(\mathrm{CC} 2 / \mathrm{X}^{\prime \prime}\right)$ scheme shown in Figure 4.3, in which TD-DFT or CIS was used both for the $\Delta \Delta E_{\text {ad }}$ and $\Delta \Delta E_{00}$ terms, the MAEs remain small even after this approximation. This was found to be particularly true for the hybrid functionals, which showed MAEs of $0.06-0.08 \mathrm{eV}$. Therefore, for a large majority of the states considered in Paper VII, it is perfectly possible to obtain CC2-quality $\Delta E_{00}$ energies by using CC2 only for the $\Delta E_{\mathrm{ve}}$ term, and employing hybrid functionals for both the $\Delta \Delta E_{\mathrm{ad}}$ and $\Delta \Delta E_{00}$ terms. 

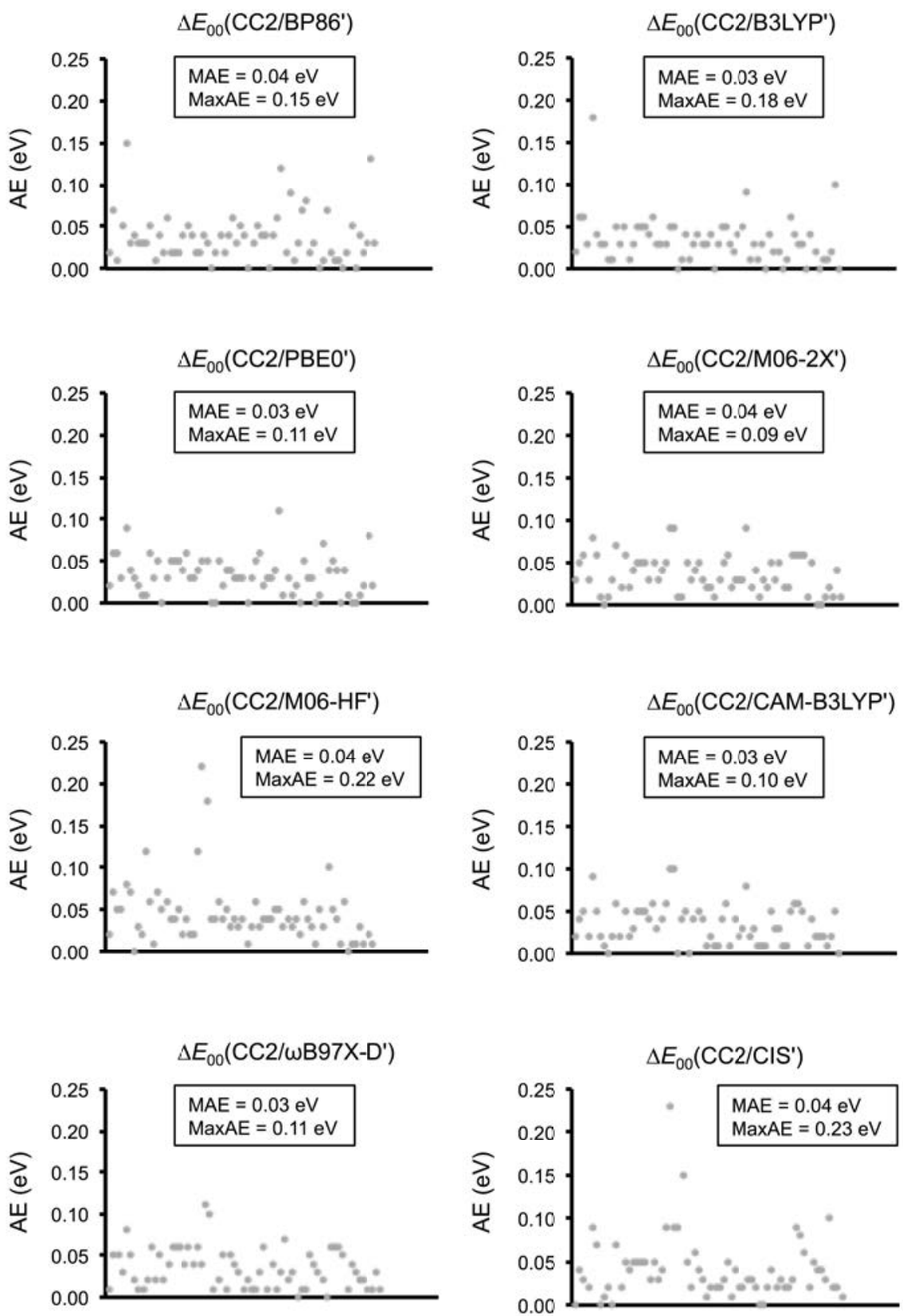

Figure 4.2. Absolute errors in calculated $\Delta E_{00}\left(\mathrm{CC} 2 / \mathrm{X}^{\prime}\right)$ energies relative to $\Delta E_{00}(\mathrm{CC} 2)$. Reprinted from Paper VII with permission from Molecular Physics. Copyright 2016 Taylor \& Francis Group Ltd. 

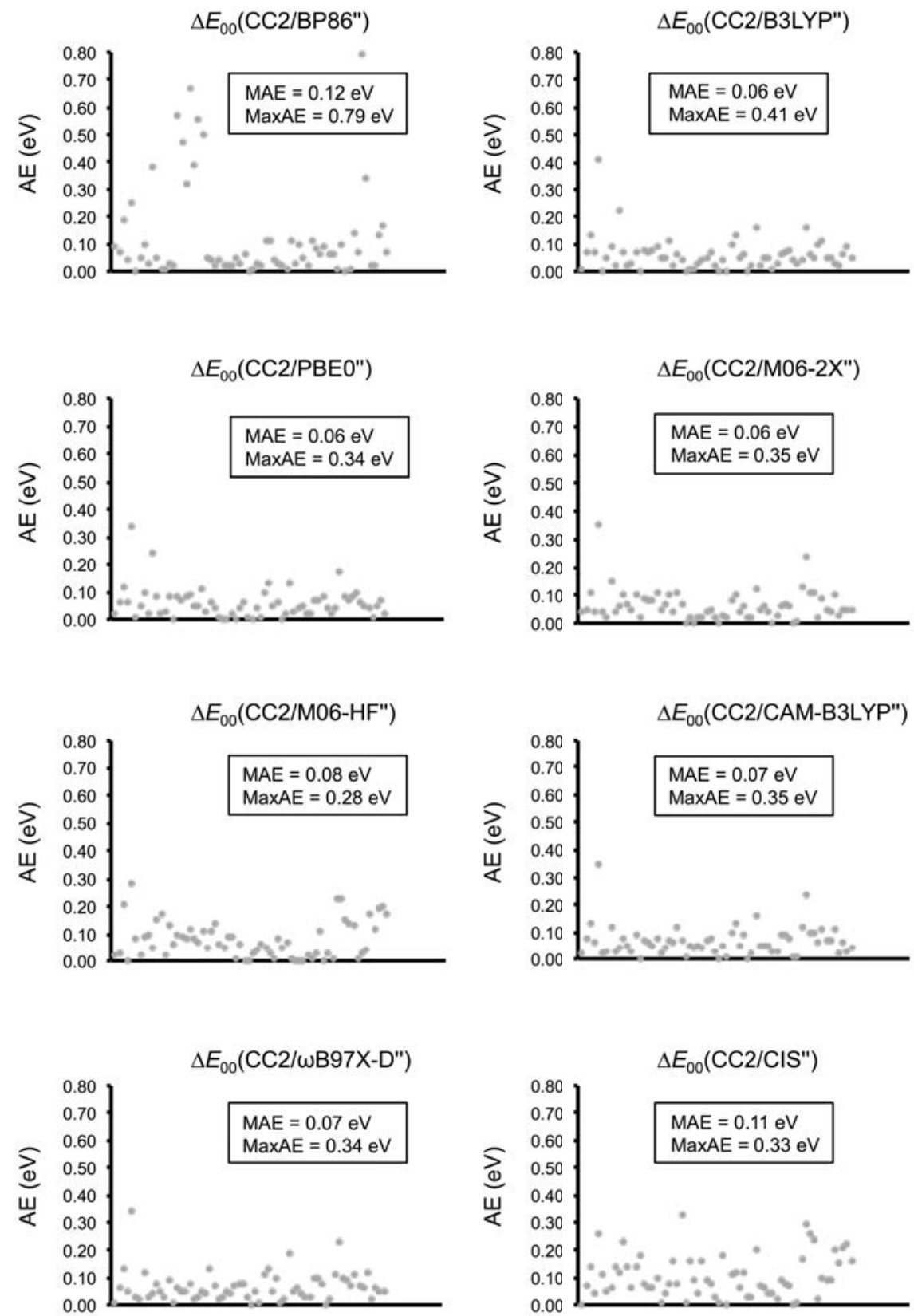

Figure 4.3. Absolute errors in calculated $\Delta E_{00}\left(\mathrm{CC} 2 / \mathrm{X}^{\prime \prime}\right)$ energies relative to $\Delta E_{00}(\mathrm{CC} 2)$. Reprinted from Paper VII with permission from Molecular Physics. Copyright 2016 Taylor \& Francis Group Ltd. 


\section{Excited-State Studies of Organic Chromophores}

The reason for the success of the composite $\Delta E_{00}\left(\mathrm{CC} 2 / \mathrm{X}^{\prime}\right)$ and $\Delta E_{00}\left(\mathrm{CC} 2 / \mathrm{X}^{\prime \prime}\right)$ schemes was found to be smaller method-sensitivity of the $\Delta \Delta E_{\mathrm{ad}}$ and $\Delta \Delta E_{00}$ energy differences compared to the $\Delta E_{\mathrm{ve}}, \Delta E_{\mathrm{ad}}$ and $\Delta E_{00}$ excitation energies. Finally, it was observed that the reason why different methods give similar $\Delta \Delta E_{\mathrm{ad}}$ relaxation energies is that they tend to provide similar excited-state geometries. 


\section{Concluding Remarks}

This thesis contains two parts. The first and major part is devoted to computational studies of two classes of light-driven molecular motors: sterically overcrowded alkenes and protonated Schiff bases. The second part is concerned with the design and assessment of a composite CC2/DFT procedure to study excited electronic states of organic chromophores.

The following conclusions emerge from computational studies of molecular motors:

- The thermal isomerizations of overcrowded alkene-based molecular motors show an intricate dependence on steric, electronic and conformational factors. By modulating these factors, several new motors capable of surpassing the thermal isomerization performance of some of the fastest available motors of this type are proposed in the thesis.

- Point chirality and (or) helical motifs that are present in almost all synthetically available light-driven molecular motors are not an absolute requirement for achieving unidirectional rotary motion. The only requirement is some kind of asymmetry in the molecular structure that breaks the symmetry between $\mathrm{CW}$ and $\mathrm{CCW}$ photoinduced rotations.

The following main conclusion emerge from the excited-state studies of organic chromophores:

- It is perfectly possible to apply composite procedures that combine $\mathrm{CC} 2$ and DFT methods for calculating $\Delta E_{00}$ energies of organic chromophores in a costeffective fashion. A prerequisite for the success of such procedures is that these methods should provide similar description of excited states. 


\section{Concluding Remarks}

Looking into the future of molecular motor research, at least three possible directions come to mind. The first is to consider other classes of molecules outside the Feringa's overcrowded-alkene design that can achieve photoinduced unidirectional rotary motion in a more efficient fashion. A step in this direction has already been taken in the thesis by considering motors incorporating protonated Schiff bases. Second, most of the light-powered motors developed to date absorb high-energy UV light, which is a drawback for biological and smart material applications. It is therefore important to design molecular systems that produce rotary motion under biocompatible conditions by absorbing either in the visible or near-IR region. Furthermore, it is also of interest to study molecular motors that are driven by sources other than light, for example, mechanically interlocked systems such as catenanes $^{143,144}$ and rotaxanes ${ }^{145,146}$ that produce chemically induced rotary motion. ${ }^{144,146}$ Jean-Pierre Sauvage and Frasser Stoddart, the co-recipients of the 2016 Nobel Prize in Chemistry, have developed effective synthetic routes to these systems. Finally, it is important to incorporate molecular motors into complex molecular devices that can accomplish useful tasks such as molecular transport.

Turning to composite procedures, a natural next step in this field would be to consider more variable types of excited states than the single-reference valence excited states considered in the thesis. Examples of such states are charge-transfer excited states. Furthermore, it would also be of interest to consider more generally applicable excited-state methods than $\mathrm{CC} 2$ such as multi-configurational quantum chemical methods. 


\section{Bibliography}

1 T. Yoshizawa and G. Wald, Nature, 1963, 197, 1279-1286.

2 G. Wald, Nature, 1968, 219, 800-807.

3 K. Kinosita Jr., K. Adachi and H. Itoh, Annu. Rev. Biophys. Biomol. Struct., 2004, 33, 245-268.

4 M. Forgac, Nat. Rev. Mol. Cell Biol., 2007, 8, 917-929.

5 R. P. Feynman, Eng. Sci., 1960, 23, 22-36.

6 N. Koumura, R. W. J. Zijlstra, R. A. van Delden, N. Harada and B. L. Feringa, Nature, 1999, 401, 152-155.

7 N. Katsonis, M. Lubomska, M. M. Pollard, B. L. Feringa and P. Rudolf, Prog. Surf. Sci., 2007, 82, 407-434.

8 G. London, G. T. Carroll and B. L. Feringa, Org. Biomol. Chem., 2013, 42, 3477-3483.

9 J. Vachon, G. T. Carroll, M. M. Pollard, E. M. Mes, A. M. Brouwer and B. L. Feringa, Photochem. Photobiol. Sci., 2014, 13, 241-246.

10 M. K. J. ter Wiel, R. A. van Delden, A. Meetsma and B. L. Feringa, J. Am. Chem. Soc., 2005, 127, 14208-14222.

11 A. C. Coleman, J. M. Beierle, M. C. A. Stuart, B. Maciá, G. Caroli, J. T. Mika, D. J. van Dijken, J. Chen, W. R. Browne and B. L. Feringa, Nat. Nanotechnol., 2011, 6, 547-552.

12 T. Kudernac, N. Ruangsupapichat, M. Parschau, B. Maciá, N. Katsonis, S. R. Harutyunyan, K. H. Ernst and B. L. Feringa, Nature, 2011, 479, 208-211.

13 P. T. Chiang, J. Mielke, J. Godoy, J. M. Guerrero, L. B. Alemany, C. J. Villagómez, A. Saywell, L. Grill and J. M. Tour, ACS Nano, 2012, 6, 592-597.

14 Q. Li, G. Fuks, E. Moulin, M. Maaloum, M. Rawiso, I. Kulic, J. T. Foy and N. Giuseppone, Nat. Nanotechnol., 2015, 10, 161-165.

15 S. J. Wezenberg, C. M. Croisetu, M. C. A. Stuart and B. L. Feringa, Chem. Sci., 2016, 7, 4341-4346.

16 D. J. van Dijken, J. Chen, M. C. A. Stuart, L. Hou and B. L. Feringa, J. Am. Chem. Soc., 2016, 138, 660-669.

17 N. Koumura, E. M. Geertsema, M. B. van Gelder, A. Meetsma and B. L. Feringa, J. Am. Chem. Soc., 2002, 124, 5037-5051.

18 M. K. J. ter Wiel, R. A. van Delden, A. Meetsma and B. L. Feringa, J. Am. Chem. Soc., 2003, 125, 15076-15086.

19 R. A. van Delden, N. Koumura, A. Schoevaars, A. Meetsma and B. L. Feringa, Org. Biomol. Chem., 2003, 1, 33-35.

20 D. Pijper, R. A. van Delden, A. Meetsma and B. L. Feringa, J. Am. Chem. Soc., 2005, 127, 17612-17613. 
22 M. M. Pollard, M. Klok, D. Pijper and B. L. Feringa, Adv. Funct. Mater., 2007, 17, 718-729.

23 M. Klok, N. Boyle, M. T. Pryce, A. Meetsma, W. R. Browne and B. L. Feringa, J. Am. Chem. Soc., 2008, 130, 10484-10485.

24 M. M. Pollard, A. Meetsma and B. L. Feringa, Org. Biomol. Chem., 2008, 6, 507-512.

25 M. M. Pollard, P. V. Wesenhagen, D. Pijper and B. L. Feringa, Org. Biomol. Chem., 2008, 6, 1605-1612.

26 R. Augulis, M. Klok, B. L. Feringa and P. H. M. van Loosdrecht, Phys. Status Solidi C, 2009, 6, 181-184.

27 T. F. Landaluce, G. London, M. M. Pollard, P. Rudolf and B. L. Feringa, J. Org. Chem., 2010, 75, 5323-5325.

28 J. Bauer, L. Hou, J. C. M. Kistemaker and B. L. Feringa, J. Org. Chem., 2014, 79, 4446-4455.

29 A. Cnossen, J. C. M. Kistemaker, T. Kojima and B. L. Feringa, J. Org. Chem., 2014, 79, 927-935.

30 J. Conyard, A. Cnossen, W. R. Browne, B. L. Feringa and S. R. Meech, J. Am. Chem. Soc., 2014, 136, 9692-9700.

31 A. Faulkner, T. van Leeuwen, B. L. Feringa and S. J. Wezenberg, J. Am. Chem. Soc., 2016, 138, 13597-13603.

32 C. Wang, H. Dong, W. Hu, Y. Liu and D. Zhu, Chem. Rev., 2012, 112, 22082267.

33 X. Guo, M. Baumgarten and K. Müllen, Prog. Polym. Sci., 2013, 38, 1832 1908.

34 J. Mei, Y. Diao, A. L. Appleton, L. Fang and Z. Bao, J. Am. Chem. Soc., 2013, 135, 6724-6746.

35 Z. Song and H. Zhou, Energy Environ. Sci., 2013, 6, 2280-2301.

36 D. F. Styer, M. S. Balkin, K. M. Becker, M. R. Burns, C. E. Dudley, S. T. Forth, J. S. Gaumer, M. A. Kramer, D. C. Oertel, L. H. Park, M. T. Rinkoski, C. T. Smith and T. D. Wotherspoon, Am. J. Phys., 2002, 70, 288-297. M. Klessinger, Angew. Chem. Int. ed., 1995, 34, 549-551.

38 F. Bernardi, M. Olivucci and M. A. Robb, Chem. Soc. Rev., 1996, 321-328.

39 D. R. Yarkony, Acc. Chem. Res., 1998, 31, 511-518.

40 D. Truhlar and C. A. Mead, Phys. Rev. A, 2003, 68, 32501.

41 B. G. Levine and T. J. Martínez, Annu. Rev. Phys. Chem., 2007, 58, 613-634.

42 M. A. Robb, in Advanced Series in Physical Chemistry: Conical Intersection Theory, Computation and Experiment, (Eds. W. Domcke, D. R. Yarkony and H. Köppel), World Scientific Publishing Co., Singapore, 2011, pp. 3-50.

43 C. C. J. Roothaan, Rev. Mod. Phys., 1951, 23, 69-89.

44 G. G. Hall, Proc. R. Soc.(London), 1951, A205, 541-552.

45 P. O. Löwdin, Phys. Rev., 1955, 97, 1509-1520.

46 B. O. Roos, P. R. Taylor and P. E. M. Siegbahn, Chem. Phys., 1980, 48, $157-$ 173.

47 T. Helgaker, P. Jørgensen and J. Olsen, Molecular electronic-structure theory, John Willey \& Sons, Chichester, 2000. 
F. Jensen, Introduction to Computational Chemistry, Second Ed., John Willey \& Sons, Chichester, 2006.

49 S. Reine and T. Saue (Eds.), European Summerschool in Quantum Chemistry 2015 Book II, 2015.

50 P. Hohenberg and W. Kohn, Phys. Rev. B, 1964, 136, B864-B871.

51 W. Kohn and L. J. Sham, Phys. Rev., 1965, 140, A1133-A1138.

52 J. P. Perdew, A. Ruzsinszky, J. Tao, V. N. Staroverov, G. E. Scuseria and G. I. Csonka, J. Chem. Phys., 2005, 123, 062201.

J. P. Perdew and K. Schmidt, AIP Conf. Proc., 2001, 577, 1-20.

54 A. D. Becke, Phys. Rev. A, 1988, 38, 3098-3100.

55 C. Lee, W. Yang and R. G. Parr, Phys. Rev. B, 1988, 37, 785-789.

56 J. P. Perdew, Phys. Rev. B, 1986, 33, 8822-8824.

57 A. D. Becke, J. Chem. Phys., 1993, 98, 1372-1377.

58 P. J. Stephens, F. J. Devlin, C. F. Chabalowski and M. J. Frisch, J. Phys. Chem., 1994, 98, 11623-11627.

59 S. Grimme, J. Chem. Phys., 2006, 124, 034108.

60 J. B. Foresman, M. Head-Gordon, J. A. Pople and M. J. Frisch, J. Phys. Chem., 1992, 96, 135-149.

61 J. Geertsen, M. Rittby and R. J. Bartlett, Chem. Phys. Lett., 1989, 164, 57-62.

62 J. F. Stanton and R. J. Bartlett, J. Chem. Phys., 1993, 98, 7029-7039.

63 O. Christiansen, H. Koch and P. Jørgensen, Chem. Phys. Lett., 1995, 243, 409418.

64 E. Runge and E. K. U. Gross, Phys. Rev. Lett., 1984, 52, 997-1000.

65 M. Petersilka, U. J. Gossmann and E. K. U. Gross, Phys. Rev. Lett., 1996, 76, 1212-1215.

66 R. Bauernschmitt and R. Ahlrichs, Chem. Phys. Lett., 1996, 256, 454-464.

67 M. E. Casida, C. Jamorski, K. C. Casida and D. R. Salahub, J. Chem. Phys., 1998, 108, 4439-4449.

68 R. E. Stratmann, G. E. Scuseria and M. J. Frisch, J. Chem. Phys., 1998, 109, 8218-8224.

69 M. A. L. Marques and E. K. U. Gross, Annu. Rev. Phys. Chem., 2004, 55, 427455.

70 A. Dreuw and M. Head-Gordon, Chem. Rev., 2005, 105, 4009-4037.

71 M. Head-Gordon, R. J. Rico, M. Oumi and T. J. Lee, Chem. Phys. Lett., 1994, 219, 21-29.

72 J. Oddershede, P. Jørgensen and D. L. Yeager, Comput. Phys. Reports, 1984, 2 , 33-92.

73 T. Yanai, D. P. Tew and N. C. Handy, Chem. Phys. Lett., 2004, 393, 51-57.

74 J.-D. Chai and M. Head-Gordon, Phys. Chem. Chem. Phys., 2008, 10, 66156620.

75 C. Van Caillie and R. D. Amos, Chem. Phys. Lett., 1999, 308, 249-255.

76 C. Van Caillie and R. D. Amos, Chem. Phys. Lett., 2000, 317, 159-164.

77 F. Furche and R. Ahlrichs, J. Chem. Phys., 2002, 117, 7433-7447.

78 M. Caricato, B. Mennucci, J. Tomasi, F. Ingrosso, R. Cammi, S. Corni and G. Scalmani, J. Chem. Phys., 2006, 124, 125420.

79 G. Scalmani, M. J. Frisch, B. Mennucci, J. Tomasi, R. Cammi and V. Barone, 
J. Chem. Phys., 2006, 124, 094107.

80 Y. Shao, M. Head-Gordon and A. I. Krylov, J. Chem. Phys., 2003, 118, 48074818.

81 L. A. Curtiss and K. Raghavachari, J. Chem. Phys., 1991, 94, 7221-7230.

82 L. A. Curtiss, K. Raghavachari and J. A. Pople, J. Chem. Phys., 1993, 98, 1293-1298.

83 L. A. Curtiss, K. Raghavachari, P. C. Redfern, V. Rassolov and J. A. Pople, J. Chem. Phys., 1998, 109, 7764-7776.

84 L. A. Curtiss, P. C. Redfern and K. Raghavachari, WIREs Comput. Mol. Sci., 2011, 1, 810-825.

85 J. M. L. Martin and G. de Oliveira, J. Chem. Phys., 1999, 111, 1843-1856.

86 A. D. Boese, M. Oren, O. Atasoylu, J. M. L. Martin, M. Kállay and J. Gauss, J. Chem. Phys., 2004, 120, 4129-4141.

87 A. Karton, E. Rabinovich, J. M. L. Martin and B. Ruscic, J. Chem. Phys., 2006, 125, 144108.

88 S. Grimme and E. I. Izgorodina, Chem. Phys., 2004, 305, 223-230.

89 P. Boulanger, D. Jacquemin, I. Duchemin and X. Blase, J. Chem. Theory Comput., 2014, 10, 1212-1218.

90 S. Chibani, A.D. Laurent, B. Le Guennic, D. Jacquemin, J. Chem. Theory Comput., 2014, 10, 4574-4582.

91 D. Jacquemin, I. Duchemin and X. Blase, J. Chem. Theory Comput., 2015, 11, 5340-5359.

92 B. Le Guennic and D. Jacquemin, Acc. Chem. Res., 2015, 48, 530-537.

93 J. Stålring, A. Bernhardsson and R. Lindh, Mol. Phys., 2001, 99, 103-114.

94 K. Andersson, P.-Å. Malmqvist, B. O. Roos, A. J. Sadlej and K. Wolinski, J. Phys. Chem., 1990, 94, 5483-5488.

95 K. Andersson, P.-Å. Malmqvist and B. O. Roos, J. Chem. Phys., 1992, 96, 1218-1226.

96 J. C. Tully, J. Chem. Phys., 1990, 93, 1061-1071.

97 S. Hammes-Schiffer and J. C. Tully, J. Chem. Phys., 1994, 101, 4657-4667.

98 T. Vreven, F. Bernardi, M. Garavelli, M. Olivucci, M. A. Robb and H. B. Schlegel, J. Am. Chem. Soc., 1997, 119, 12687-12688.

99 O. Weingart, A. Migani, M. Olivucci, M. A. Robb, V. Buss and P. Hunt, $J$. Phys. Chem. A, 2004, 108, 4685-4693.

100 G. Groenhof, M. Bouxin-Cademartory, B. Hess, S. P. de Visser, H. J. C. Berendsen, M. Olivucci, A. E. Mark and M. A. Robb, J. Am. Chem. Soc., 2004, 126, 4228-4233.

101 G. Groenhof, L. V. Schäfer, M. Boggio-Pasqua, M. Goette, H. Grubmüller and M. A. Robb, J. Am. Chem. Soc., 2007, 129, 6812-6819.

102 E. Fabiano, G. Groenhof and W. Thiel, Chem. Phys., 2008, 351, 111-116.

103 M. Barbatti, WIREs Comput. Mol. Sci., 2011, 1, 620-633.

104 E. Tapavicza, G. D. Bellchambers, J. C. Vincent and F. Furche, Phys. Chem. Chem. Phys., 2013, 15, 18336-18348.

105 I. F. Galván, M. G. Delcey, T. B. Pedersen, F. Aquilante and R. Lindh, J. Chem. Theory Comput., 2016, 12, 3636-3653.

106 A. Warshel and M. Levitt, J. Mol. Biol., 1976, 103, 227-249. 
107 H. Lin and D. G. Truhlar, Theor. Chem. Acc., 2007, 117, 185-199.

108 H. M. Senn and W. Thiel, Angew. Chem. Int. Ed., 2009, 48, 1198-1229.

109 B. Mennucci, E. Cancès and J. Tomasi, J. Phys. Chem. B, 1997, 101, 1050610517.

110 E. Cancès, B. Mennucci and J. Tomasi, J. Chem. Phys., 1997, 107, 3032-3041.

111 C. J. Cramer and D. G. Truhlar, Chem. Rev., 1999, 99, 2161-2200.

112 A. V Marenich, C. J. Cramer and D. G. Truhlar, J. Phys. Chem. B, 2009, 113, 6378-6396.

113 M. Guentner, M. Schildhauer, S. Thumser, P. Mayer, D. Stephenson, P. J. Mayer and H. Dube, Nat. Commun., 2015, 6, 8406.

114 J. C. M. Kistemaker, P. Štacko, J. Visser and B. L. Feringa, Nat. Chem., 2015, 7, 890-896.

115 L. Greb and J.-M. Lehn, J. Am. Chem. Soc., 2014, 136, 13114-13117.

116 L. Greb, A. Eichhöfer and J.-M. Lehn, Angew. Chem. Int. Ed., 2015, 54, 14345-14348.

117 C. Adamo and V. Barone, J. Chem. Phys., 1999, 110, 6158-6170.

118 Y. Zhao and D. G. Truhlar, Theor. Chem. Acc., 2008, 120, 215-241.

119 S. Grimme, J. Comput. Chem., 2004, 25, 1463-1473.

120 J. Antony and S. Grimme, Phys. Chem. Chem. Phys., 2006, 8, 5287-5293.

121 J. Tittor and D. Oesterhelt, FEBS Lett., 1990, 263, 269-273.

122 R. W. Schoenlein, L. A. Peteanu, R. A. Mathies and C. V. Shank, Science, 1991, 254, 412-415.

123 Q. Wang, R. W. Schoenlein, L. A. Peteanu, R. A. Mathies and C. V. Shank, Science, 1994, 266, 422-424.

124 H. Kandori, Y. Shichida and T. Yoshizawa, Biochem., 2001, 66, 1197-1209.

125 A. Kazaryan, Z. Lan, L. V. Schäfer, W. Thiel and M. Filatov, J. Chem. Theory Comput., 2011, 7, 2189-2199.

126 F. Lumento, V. Zanirato, S. Fusi, E. Busi, L. Latterini, F. Elisei, A. Sinicropi, T. Andruniów, N. Ferré, R. Basosi and M. Olivucci, Angew. Chem. Int. Ed., 2007, 46, 414-420.

127 G. Marchand, J. Eng, I. Schapiro, A. Valentini, L. M. Frutos, E. Pieri, M. Olivucci, J. Léonard and E. Gindensperger, J. Phys. Chem. Lett., 2015, 6, 599604.

128 B. Moore II, A. Charaf-Eddin, A. Planchat, C. Adamo, J. Autschbach and D. Jacquemin, J. Chem. Theory Comput., 2014, 10, 4599-4608.

129 T. Petrenko and F. Neese, J. Chem. Phys., 2007, 127, 164319.

130 F. J. A. Ferrer, J. Cerezo, E. Stendardo, R. Improta and F. Santoro, J. Chem. Theory Comput., 2013, 9, 2072-2082.

131 E. Kamarchik and A. I. Krylov, J. Phys. Chem. Lett., 2011, 2, 488-492.

132 M. Dierksen and S. Grimme, J. Chem. Phys., 2004, 120, 3544-3554.

133 M. Dierksen and S. Grimme, J. Phys. Chem. A, 2004, 108, 10225-10237.

134 M. Uppsten and B. Durbeej, J. Comput. Chem., 2012, 33, 1892-1901.

135 R. Send, M. Kühn and F. Furche, J. Chem. Theory Comput., 2011, 7, 23762386.

136 D. Jacquemin, A. Planchat, C. Adamo and B. Mennucci, J. Chem. Theory Comput., 2012, 8, 2359-2372. 
137 L. Barnes, S. Abdul-Al and A. R. Allouche, J. Phys. Chem. A, 2014, 118, 11033-11046.

138 J. F. Stanton and J. Gauss, J. Chem. Phys., 1994, 101, 8938-8944.

139 A. Köhn and C. Hättig, J. Chem. Phys., 2003, 119, 5021-5036.

140 S. V. Levchenko, T. Wang and A. I. Krylov, J. Chem. Phys., 2005, 122, 224106.

141 M. G. Delcey, L. Freitag, T. B. Pedersen, F. Aquilante, R. Lindh and L. González, J. Chem. Phys., 2014, 140, 174103.

142 Y. Zhao and D. G. Truhlar, J. Phys. Chem. A, 2006, 110, 13126-13130.

143 C. O. Dietrich-Buchecker, J. P. Sauvage and J. P. Kintzinger, Tetrahedron Lett., 1983, 24, 5095-5098.

144 M. R. Wilson, J. Solà, A. Carlone, S. M. Goldup, N. Lebrasseur and D. A. Leigh, Nature, 2016, 534, 235-240.

145 P. L. Anelli, N. Spencer and J. F. Stoddart, J. Am. Chem. Soc., 1991, 113, 5131-5133.

146 D. A. Leigh, J. K. Y. Wong, F. Dehez and F. Zerbetto, Nature, 2003, 424, 174 179. 


\section{Publications Included in the Thesis}

I Computational Study of the Working Mechanism and Rate Acceleration of Overcrowded Alkene-Based Light-Driven Rotary Molecular Motors

C. Fang, B. Oruganti and B. Durbeej RSC Adv., 2014, 4, 10240-10251.

II Computational Design of Faster Rotating Second-Generation LightDriven Molecular Motors by Control of Steric Effects

B. Oruganti,* C. Fang* and B. Durbeej *Co-first authorship Phys. Chem. Chem. Phys., 2015, 17, 21740-21751.

III On the Possibility to Accelerate the Thermal Isomerizations of Overcrowded Alkene-Based Rotary Molecular Motors with ElectronDonating or Electron-Withdrawing Substituents

B. Oruganti and B. Durbeej

J. Mol. Model., 2016, 22, 219.

IV Computational Insight to Improve the Thermal Isomerisation Performance of Overcrowded Alkene-Based Molecular Motors through Structural Redesign

B. Oruganti, J. Wang and B. Durbeej

ChemPhysChem, 2016, DOI: 10.1002/cphc.201600766.

V Light-Driven Unidirectional Rotary Motion in Molecular Motors Lacking Point Chirality and Helical Motifs

J. Wang, ${ }^{*}$ B. Oruganti* and B. Durbeej $\quad *$ Co-first authorship

Submitted to J. Phys. Chem. Lett.

VI How Method-Dependent are Calculated Differences between Vertical, Adiabatic, and 0-0 Excitation Energies?

C. Fang, B. Oruganti and B. Durbeej

J. Phys. Chem. A, 2014, 118, 4157-4171. 


\section{Assessment of a Composite CC2/DFT Procedure for Calculating 0-0 Excitation Energies of Organic Molecules \\ B. Oruganti, * C. Fang* and B. Durbeej *Co-first authorship Mol. Phys., 2016, DOI: 10.1080/00268976.2016.1235736.}

\section{My contributions to the papers}

Paper I: I planned the research together with Bo Durbeej and Changfeng Fang, performed calculations together with Changfeng Fang, and wrote the manuscript together with both co-authors.

Paper II: I planned the research together with Bo Durbeej and Changfeng Fang, performed calculations together with Changfeng Fang, and wrote the manuscript together with both co-authors.

Paper III: I planned the research together with Bo Durbeej, performed all calculations, and wrote the manuscript together with Bo Durbeej.

Paper IV: I planned the research together with Bo Durbeej, performed calculations together with Jun Wang, and wrote the manuscript together with Bo Durbeej.

Paper V: I planned the research together with Bo Durbeej and Jun Wang, performed calculations together with Jun Wang, and wrote the manuscript together with both coauthors.

Paper VI: I planned the research together with Bo Durbeej and Changfeng Fang, performed calculations together with Changfeng Fang, and wrote the manuscript together with both co-authors.

Paper VII: I planned the research together with Bo Durbeej and Changfeng Fang, performed calculations together with Changfeng Fang, and wrote the manuscript together with both co-authors. 


\section{Publications}

The articles associated with this thesis have been removed for copyright reasons. For more details about these see:

http://urn.kb.se/resolve?urn=urn:nbn:se:liu:diva-132611 